\title{
ARTICLE
}

Received 10 Jul 2014 | Accepted 10 Oct 2014 | Published 24 Nov $2014 \quad$ DOl: 10.1038/ncomms6549

\section{Regulation of Drosophila circadian rhythms by miRNA let-7 is mediated by a regulatory cycle}

Wenfeng Chen ${ }^{1, \star}$, Zhenxing Liu ${ }^{1, \star}$, Tianjiao $\mathrm{Li}^{1}$, Ruifeng Zhang ${ }^{1}$, Yongbo Xue ${ }^{1}$, Yang Zhong ${ }^{1}$, Weiwei Bai', Dasen Zhou ${ }^{1} \&$ Zhangwu Zhao ${ }^{1}$

MicroRNA-mediated post-transcriptional regulations are increasingly recognized as important components of the circadian rhythm. Here we identify microRNA let-7, part of the Drosophila let-7-Complex, as a regulator of circadian rhythms mediated by a circadian regulatory cycle. Overexpression of let-7 in clock neurons lengthens circadian period and its deletion attenuates the morning activity peak as well as molecular oscillation. Let-7 regulates the circadian rhythm via repression of CLOCKWORK ORANGE (CWO). Conversely, upregulated cwo in cwo-expressing cells can rescue the phenotype of let-7-Complex overexpression. Moreover, circadian prothoracicotropic hormone (PTTH) and CLOCKregulated $20-\mathrm{OH}$ ecdysteroid signalling contribute to the circadian expression of let-7 through the $20-\mathrm{OH}$ ecdysteroid receptor. Thus, we find a regulatory cycle involving PTTH, a direct target of CLOCK, and PTTH-driven miRNA let-7.

\footnotetext{
${ }^{1}$ Department of Entomology, College of Agronomy and Biotechnology, China Agricultural University, Beijing 100193, China. * These authors contributed equally to this work. Correspondence and requests for materials should be addressed to Z.Z. (email: zhaozw@cau.edu.cn).
} 
A lmost all animals display a wide range of circadian rhythms in behaviour and physiology, such as locomotor activity, sleep, learning and memory, mating and endocrine function ${ }^{1-4}$. The circadian system includes an input pathway, a core clock system and an output pathway ${ }^{5}$. Through the input pathway, a circadian oscillator receives external information, such as light and temperature signals, that entrain the core oscillator 6 . The core clock system is an integrative oscillator for a self-sustaining rhythm, and the output pathways transmit signals from the oscillator to observable rhythms in behaviour and physiology.

Numerous studies have focused on the molecular mechanisms and neural network properties of the circadian oscillator. The positive transcriptional regulatory factors CLOCK (CLK) and CYCLE (CYC) form heterodimers that lead to the transcription of the negative regulators PERIOD (PER) and TIMELESS (TIM) via binding to E-boxes in their promoters ${ }^{7,8}$. PER and TIM proteins accumulate in the cytoplasm with a delay and enter the nucleus to repress CLK-CYC-mediated transcription by collaborating with other factors like kinases ${ }^{9}$. CLK-CYC also activates two other feedback loops critical for the phase and amplitude of core oscillators and outputs. One feedback loop affecting Clk mRNA cycling is mediated by VRILLE (VRI) and PAR DOMAIN PROTEIN 1 expression ${ }^{10,11}$, and another is mediated by CLOCKWORK ORANGE (CWO), which is activated by CLK$\mathrm{CYC}^{12-15}$. The per and tim genes receive two opposing signals form DNA-binding transcription factors-a direct activation from CLK-CYC and a direct repression from CWO. In addition, CWO regulates the level of the CLK-CYC, presumably through its actions on each of three main loops ${ }^{16}$. So cwo contributes to sustaining a high-amplitude circadian oscillation in vivo ${ }^{12-14}$. The Drosophila rhythm strength is sensitive to the level and timing of cwo expression, although the amplitude of the cwo mRNA oscillation is much weaker than that of the other core clock genes $^{15}$.

In the adult fly brain, $\sim 150$ clock neurons are organized into six major clusters: three dorsal clusters (DN1, DNs and DN3), a dorsal-lateral cluster and two groups of ventral-lateral neurons (sLNvs and lLNvs) ${ }^{17}$. Under light-dark (LD) conditions, flies show a morning activity peak (M) around the time of lights-on and an evening activity peak (E) around the time of lights-off. Flies can increase their locomotor activity in advance of both dark-to-light and light-to-dark transitions-a phenomenon termed anticipation. The morning and evening anticipatory behaviour is generated in different clusters of clock neurons ${ }^{18,19}$.

Beyond the abundant information on the transcriptionaltranslation feedback loops of circadian rhythms, the importance of post-transcriptional mechanisms is increasingly recognized in circadian rhythms ${ }^{20,21}$, such as regulation by microRNAs (miRNAs) ${ }^{22}$. The miRNAs are endogenous small non-coding RNAs (19-25 nt) that function in post-transcriptional regulation of gene expression, and are found to target more than $30 \%$ of all protein-coding mRNAs in mammals ${ }^{23,24}$. Via base-pairing with specific sequences of target genes typically found in mRNA $3^{\prime}$ untranslated regions ( $3^{\prime}$ UTRs), they usually result in gene silencing via translational repression or target mRNA degradation by an RNA-induced silencing complex consisting of Argonaute catalytic subunits (Agol in Drosophila) ${ }^{25,26}$. One requirement for specificity is an accurate match between the $5^{\prime}$-proximal 'seed' region of the miRNA and the $3^{\prime}$ UTRs of its target mRNA ${ }^{27}$. Previous studies have revealed that miRNAs are involved in regulation of the core circadian oscillator and the clock outputs in Drosophila. By immunoprecipitating mRNAs bound to AGO1, Kadener et al. ${ }^{28}$ found that core clock genes Clk, Vri and Cwo are associated with RNA-induced silencing complex, and that bantam-dependent regulation of $C l k$ expression is required for circadian rhythm. Two miRNAs (miR-263a and -263b) were also found to exhibit robust daily changes in abundance in wild-type flies that are abolished in the $c y c^{01}$ mutants $^{29}$. In addition, the miR-279 influences circadian behavioural outputs via targeting upd, a ligand of the JAK/STAT pathway ${ }^{30}$, and the oscillating miRNA 959-964 cluster impacts Drosophila feeding time and circadian outputs ${ }^{31}$.

To further investigate the role of miRNAs in the Drosophila core circadian clock, we focus on the core clock gene cwo because it is rhythmically expressed and contributes to a high-amplitude circadian oscillation of core clock genes in vivo. Drosophila rhythm strength is sensitive to cwo both in expression level and timing $^{12-14}$. Here we report that the miRNA let-7 affects the locomotor rhythm through the circadian-relevant target gene cwo in the central clock. We also study the signal-transduction pathway and molecular mechanism by which let-7 regulates the daily circadian rhythm, and show that let-7 is involved in an ecdysteroid-signalling cycle.

\section{Results}

Overexpression of let-7 prolongs activity rhythms. Because the expression of the let-7-Complex (let-7-C) or let-7 driven by the tim-GAL4 led to embryonic lethality, we used a $p d f$-Gal4 driver to overexpress let-7-C or let-7 in the pdf-expressing cells (LNvs), which control locomotor activity rhythms. Let-7-C or let-7 overexpression driven by $p d f$-GAL4 lengthened circadian period about 2 and $1 \mathrm{~h}$, respectively. In flies expressing mutant let-7 (UAS-mut-let-7), which was generated by site-directed mutagenesis of the UAS-let-7 construct, with G-C transitions at seed sequence sites 3-4 of the mature let-7 miRNA and complementary mutations on the opposing side of the hairpin ${ }^{32}$ (Supplementary Fig. 1a), the circadian period was normal (Table 1 and Fig. 1a). These results indicate that let-7 affects circadian period. To exclude the possibility that the behavioural phenotype was caused by ectopic induction of let-7 in neurons where it is usually not expressed, we utilized a knock-in Gal4 in the let-7-C locus (Supplementary Fig. $1 \mathrm{~b})^{33}$ to drive expression of let-7-C, and showed the miRNA levels of let-7, miR-125 and miR-100 were more abundant (Supplementary Fig. 2a). Overexpression of let-7-C driven by let-7-C-Gal4 phenocopies the effect driven by $p d f$-Gal4 (Fig. 1a and Table 1), suggesting that let-7 regulates circadian rhythms in regions of its normal expression. To distinguish effects of let-7 in adult and in developmental processes, we used a temperature-sensitive tubulin-Gal80 ts coupled with the tim-Gal4 driver to overexpress let-7-C, let-7 and miR-125 in adults. Results showed that the miRNA levels of let-7 and miR-125 were more abundant compared to control (Supplementary Fig. 2b). Adult-specific overexpression of let-7-C and let-7 in tim cells resulted in longer circadian periods (Fig. 1b) and lower percentages of rhythmic flies when flies were shifted from 18 to $29^{\circ} \mathrm{C}$. Circadian behavioural rhythms were present in $58.1 \%(\tau=25.2 \mathrm{~h}, n=43)$ and $77.8 \%(\tau=25.0 \mathrm{~h}, n=45)$ of the let-7-C and let-7 flies, respectively, in constant darkness (DD), while control flies exhibited normal behavioural rhythms (85.1\% rhythmic, $\tau=23.8 \mathrm{~h}, \quad n=47)$. By contrast, the flies with miR-125 overexpression and control flies exhibited similar behavioural rhythm (87.8\% rhythmic, $\tau=24.3 \mathrm{~h}, n=49)$. The results show that let-7 affects the central clock. We further drove let-7-C expression with a drug (RU486) inducible pan-neuronal driver (elavGeneSwitch; elavGS), which also lengthened period in DD (Fig. 1c). Taken together, all of these results demonstrate that adult-specific overexpression of let-7 in circadian cells lengthens circadian period and reduces rhythmicity. 
Table 1 | Locomotor activity of flies with altered let-7 levels.

\begin{tabular}{|c|c|c|c|c|}
\hline Genetype & Total flies & Rhythmic flies (\%) & Period (h) & Power \\
\hline pdf-Gal4/+; UAS-let-7-C/+ & 80 & 95.0 & $26.12 \pm 0.24$ & $82.2 \pm 2.89$ \\
\hline pdf-Gal4/ + & 61 & 100 & $24.02 \pm 0.20$ & $98.3 \pm 4.24$ \\
\hline UAS-let-7-C/+ & 51 & 95.2 & $23.86 \pm 0.52$ & $74.3 \pm 5.13$ \\
\hline let-7-C-Gal4/+ ; UAS-let-7-C/+ & 53 & 90.0 & $25.04 \pm 0.26$ & $79.7 \pm 5.74$ \\
\hline let-7-C-Gal4/+(let-7-CGKI/+) & 60 & 97.0 & $23.89 \pm 0.05$ & $113.5 \pm 3.28$ \\
\hline pdf-Gal4/+ ; UAS-let-7/+ & 31 & 100 & $24.98 \pm 0.04$ & $80.1 \pm 5.29$ \\
\hline pdf-Gal4/+ ; UAS-mut-let-7/+ & 39 & 99.0 & $23.14 \pm 0.21$ & $85.6 \pm 5.63$ \\
\hline let-7-CKO1/GKI & 48 & 50.0 & $23.75 \pm 0.18$ & $58.5 \pm 4.34$ \\
\hline let-7-CKO1/+ & 47 & 91.0 & $23.71 \pm 0.04$ & $97.7 \pm 5.21$ \\
\hline$P\{W 8$, let-7-C\}/Y; let-7-CKO1/GKI & 56 & 100 & $24.79 \pm 0.07$ & $89.2 \pm 6.27$ \\
\hline$P\{W 8$, let-7-C $\} / Y$; let-7-CKO1/KO1 & 47 & 92.0 & $24.36 \pm 0.06$ & $75.2 \pm 6.30$ \\
\hline$y w ; \triangle l e t-7, \operatorname{miR}-125$ & 42 & 48.0 & $24.20 \pm 0.20$ & $52.3 \pm 5.84$ \\
\hline$y w ; \triangle l e t-7, \operatorname{miR}-125 /+$ & 36 & 90.0 & $23.90 \pm 0.06$ & $94.2 \pm 9.97$ \\
\hline
\end{tabular}

Let-7 overexpression in clock neurons maintain the normal rhythm. Both let-7-C and let-7 mutants become largely arrhythmic in constant darkness (DD). Flies regain the normal rhythm by inserting the $P$ element $-P\{W 8$, let-7- $C\}$ into let-7-C mutants.

Let-7 is required for normal circadian rhythms. Deletions of the let-7-C and let-7,miR-125 had been generated by homologous recombination methods ${ }^{32,33}$. Single let-7, miR-125, miR-100 mutant strains were generated by deletions of rescuing transgenes, in which the expression of let-7, $m i R-100$ or $m i R-125$ had been separately eliminated, introduced into strains with deletions of the entire endogenous let-7-C locusnamed $\triangle$ let-7 (KO1/GKI;pP\{W8,let-7-C $\left.\left.C^{\triangle l e t-7}\right\} /+\right), \triangle m i R-100$ $\left(\right.$ KO1/GKI;pP $\left\{W 8\right.$,let-7-C $\left.\left.C^{\triangle m i R-100}\right\} /+\right)$ and $\triangle m i R-125$ (KO1/ GKI;pP\{W8,let-7-C $\left.C^{\triangle m i R-125}\right\} /+$ ) (Supplementary Fig. 1b) ${ }^{33}$. In this study, PCR and real time quantitative reverse transcriptase PCR analysis were performed to confirm the deletion of the let-7-C locus and individual miRNAs (Supplementary Fig. 2c,d).

To test whether the let-7 is required for normal circadian rhythmicity, we analysed the locomotor activity in different let-7-C mutants. Results showed that there was no anticipation of lights-on in let-7-C mutants (KO1/GKI) under LD, and this phenotype was conspicuously different from that of wild-type and let-7-C mutant heterozygous flies $(K O 1 /+\& G K I /+)$ (Fig. 2). The $\triangle m i R-100$ mutants exhibited normal morning and evening peaks, suggesting that $m i R-100$ was not responsible for the circadian defect. In contrast, $\triangle$ let-7,miR-125 mutants exhibited severely suppressed morning peaks before light-on. The absence of lights-on anticipation was also observed in $\triangle$ let- 7 mutants but not in $\triangle m i R-125$ mutants (Fig. 2), indicating that let-7 is responsible for the circadian defect in morning anticipation in let-7-C mutants, which could be well rescued by UAS-let-7 in pigment dispersing factor (PDF) neurons (Fig. 2). Because the lights-on response in $\mathrm{LD}$ can mask some of the clock-driven morning behaviour ${ }^{34}$, the first day of DD after LD entrainment was also assessed, and this analysis confirmed the lack of morning anticipation in the let-7 mutants (Fig. 2). Besides, both let-7-C and $\triangle$ let-7,miR-125 mutants became largely arrhythmic in DD (both produced about $50 \%$ rhythmic flies), indicating that let-7 is also required for robust $24 \mathrm{~h}$ free-running rhythms (Table 1 ).

CLOCKWORK ORANGE (cwo) is a target of let-7. The Drosophila Cwo gene was identified by bioinformatics as a possible target for let-7 (refs 35,36) (Supplementary Fig. 3). Cwo produces three mRNAs that encode two different proteins, in which all of the transcripts contain the same $\sim 1.4-\mathrm{kb} 3^{\prime}$ UTR. Detection of AGO1-associated mRNAs from fly heads also showed that Cwo might be a miRNA-regulated target ${ }^{28}$. To test whether let-7 can actually regulate the Cwo $3^{\prime}$ UTR, S2 cells were transfected to express the $3^{\prime}$ UTR of Cwo downstream of a luciferase reporter.
The cells were also separately transfected to express pri-let-7 (long let-7 precursor). Results showed that the let-7 reduced activity of the luciferase $\sim 70 \%$, and mutation of the let-7 target sites in the seed regions of the Cwo $3^{\prime} \mathrm{UTR}$ produced a recovery of activity compared to control (Fig. 3a). The results in let-7-transfected S2 cells showed that let-7 expression directly reduced the Cwo mRNA and protein levels (Fig. 3b). Moreover, AGO1-associated cwo mRNA from fly heads of wild-type and the $\triangle$ let-7 mutant using the AGO1 IP method of Kadener et al. ${ }^{28}$ were also assayed. Results showed that cwo mRNA from AGO1 immunoprecipitates was greatly diminished in $\triangle$ let-7 mutant (Fig. 3c). All these results support that cwo is a direct target of let-7.

Does downregulation of Cwo by let-7 contribute to the normal circadian rhythm? To explore this question, we altered the Cwo level by using UAS-let-7-C driven by cwo-GAL4. Results showed that let-7-C overexpression driven by cwo-GAL4 (cwo-Gal4/UASlet-7-C) lengthened circadian period (Fig. 3d), which is consistent with the effect of $c w o-R N A i$ (long circadian period) driven by both cwo-Gal4 and tim-Gal4 (Fig. 3d), and it was able to be partially rescued by Cwo overexpression (UAS-cwo/ + ; cwo-Gal4/ UAS-let-7-C) (Fig. 3d and Supplementary Fig. 4). Let-7 expression in cwo neurons (cwo-Gal4/UAS-let-7) resulted in complete lethality. In order to verify that the lethality is due to the reduction of $C w o$, we also performed a genetic rescue experiment, in which the viability was normal in UAS-cwo/ + ; cwo-Gal4/UASlet-7 transgenic flies compared to controls (Supplementary Fig. 5). Taken together, all these findings demonstrate that miRNA let-7mediated Cwo regulation contributes to the circadian rhythm.

Let-7 regulates pacemaker neurons. Because let-7 appears to function in the central clock, we determined whether let-7 is expressed in the central pacemaker by labelling let-7-C-expressing neurons (let-7-C-GAL4 $\times U A S-E G F P)$ with green fluorescent protein (GFP) and immunolabeling with anti-PDF antibody. Results showed that let-7 was broadly expressed in the fly brain. The GFP signal was enriched in brain regions such as the mushroom bodies (Fig. 4a). The co-detection in brains for GFP and PDF revealed that let-7-C was expressed in the LNvs (Fig. 4a). The presence of a GFP signal in clock neurons in let-7-C-GAL4:UAS-EGFP flies further supports the action of let-7 in the central pacemaker. To further investigate the expression of let-7 and its target Cwo in the brain, we also analysed two transcriptional reporters that contain almost the entire first intron of let-7-C (let-7-Cp12.5kb::lacZ) or just the $20-\mathrm{OH}$ ecdysteroid (20E)-responsive $3.3 \mathrm{~kb}$ fragment (let-7-Cp3.3kb::lacZ) with 


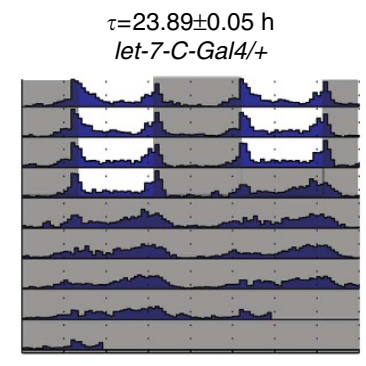

$\tau=25.04 \pm 0.26 \mathrm{~h}$

let-7-C-Gal4/+;UAS-let-7-C/+

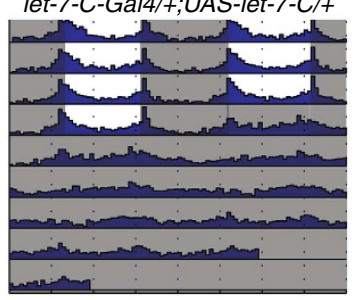

$\tau=24.0 \pm 0.15 \mathrm{~h}$

UAS-let-7-C/+

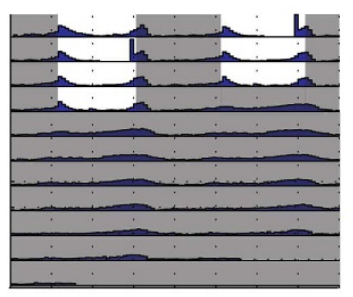

$\tau=26.12 \pm 0.24 \mathrm{~h}$

pdf-Gal4/+;UAS-let-7-C/+

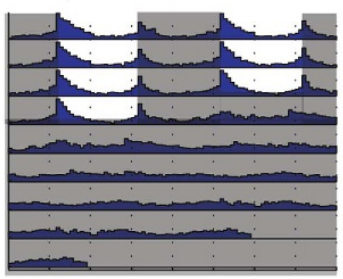

$\tau=24.02 \pm 0.20 \mathrm{~h}$ pdf-Gal4/+

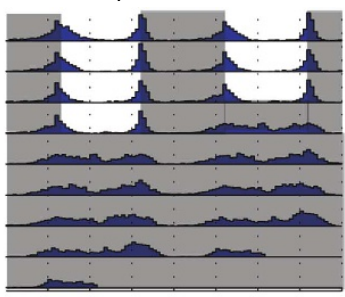

$\tau=24.98 \pm 0.04 \mathrm{~h}$ pdf-Gal4/+;UAS-let-7/+

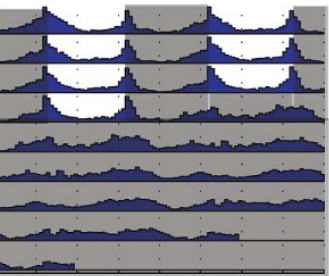

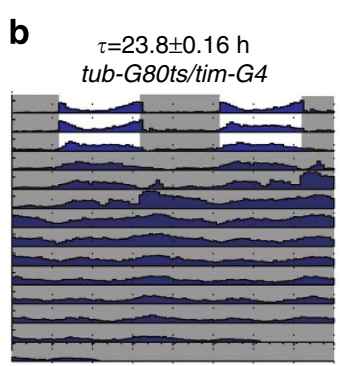
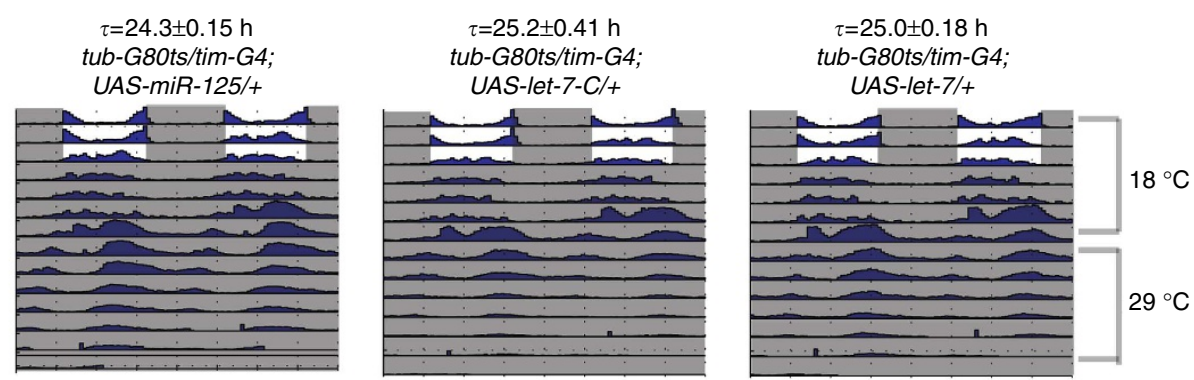

C

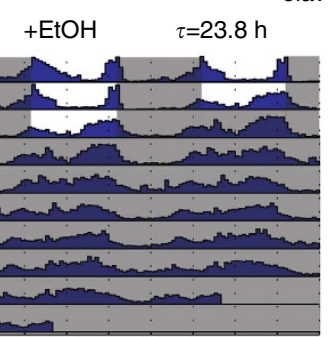

elavGS/+

elavGS/UAS-let-7
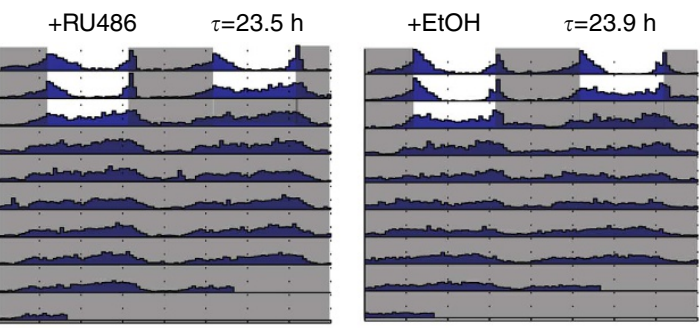

$+\mathrm{RU} 486$

$\tau=27.3 \mathrm{~h}$

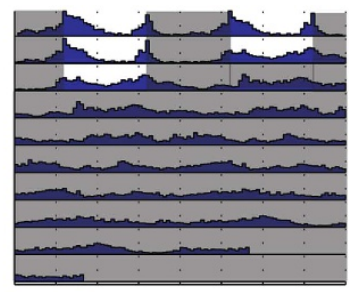

Figure 1 | Overexpression of let-7 influences locomotor activity rhythms. (a) Overexpression of let-7-C or let-7 with let-7-C-Gal4 and pdf-Gal4 leads to long behavioural periods in DD. The genotypes are indicated on top of the panels. The white and grey parts indicate light and dark, respectively. (b) Induction of let-7 in tim cells leads to long behavioural periods in DD. To restrict expression of let-7 in adults, tim-Gal4-induced expression was repressed during development by a tubulin-GAL8O ${ }^{\text {ts }}$. Flies were reared and entrained for 3 days following eclosion at $18^{\circ} \mathrm{C}$, free-running activity rhythms were monitored for 3 days at $18^{\circ} \mathrm{C}$, and then the temperature was shifted to $29^{\circ} \mathrm{C}$. The genotypes are indicated on the top of the panels. The white and grey parts indicate light and dark, respectively. (c) Pan-neuronal induction of let-7 in adulthood leads to a long period. Files were reared and then aged for 3 days following eclosion on regular food. They were fed either $500 \mu \mathrm{M}$ RU486 or ethanol (vehicle control) from the time of entrainment. Average periods of the DD records are shown. The white and gray parts indicate day and night, respectively. Each experiment was conducted at least three times, and 60-90 flies of each genotype were used for behavior assays.

cwo-Gal4 driving $\mathrm{GFP}^{37}$. The Cwo reporter was prominently expressed in the LNvs, the PI neurons and the SG (Fig. 4b). The spatial expression of lacZ resembled that previously reported in let-7-C-Gal4:UAS-EGFP. All of the reporters exhibit coexpression of let-7-C in the LNv neurons.

To test whether let-7 affects the central clock, we explored its effects on the daily expression of CWO proteins. Temporal expression profiles of CWO protein in wild-type and let-7-C mutant and $\triangle$ let-7 mutant flies under LD conditions showed that CWO was highly expressed in these two mutant fly heads compared to wild-type flies (Fig. 5). In addition, as shown in Fig. 6, let-7-C mutant flies and wild-type controls do not exhibit similar daily PER and PDF cycling in the central clock tissue, in which PDF is higher and nuclear PER accumulates sooner in let-7-C mutant flies due to a decrease of let-7, without an effect on the time of peak nuclear PER accumulation. These results above indicate that let-7 has a function in the core clock through CWO.

A novel regulatory cycle in circadian rhythm. To test whether let-7 itself is regulated by the circadian clock, we further assayed let-7 in wild-type flies and $\mathrm{Clk}^{\text {rrk }}$ mutants and found that the expression of mature let-7 exhibited statistically significant (albeit 


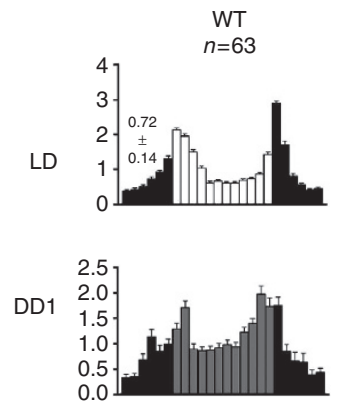

KO1/GKI

$n=88$
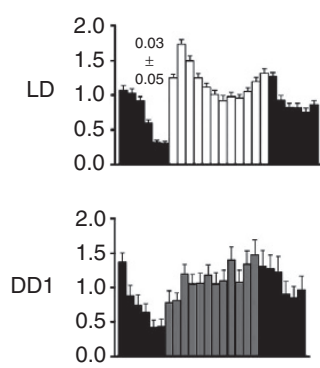
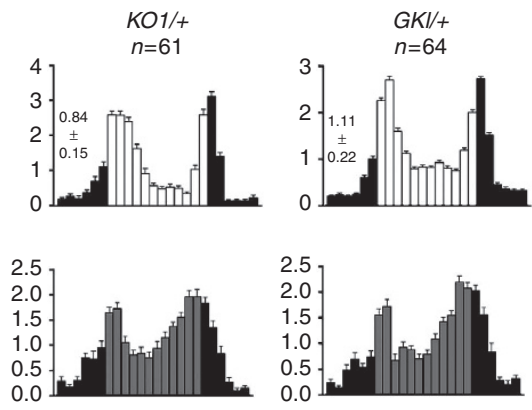

yw; 4 let-7, $\operatorname{miR}-125$ $n=32$
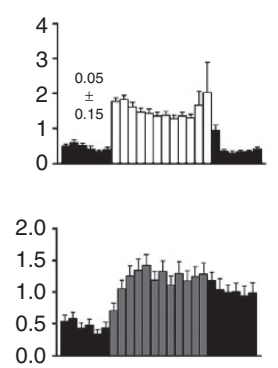

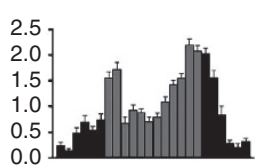

KO1/GKl; $p P$ $n=82$
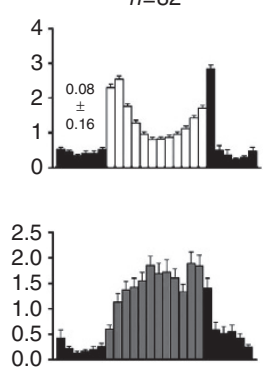
$\left\{W 8\right.$, let-7-C $\left.C^{4 l e t-7}\right\} /+$
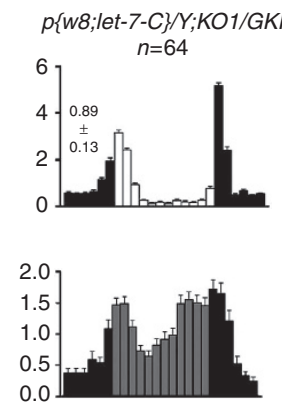

KO1/GKl; $p P$ $\left\{W 8\right.$, let-7-C $\left.C^{\Delta \operatorname{miR}-125}\right\} /+$ $n=83$
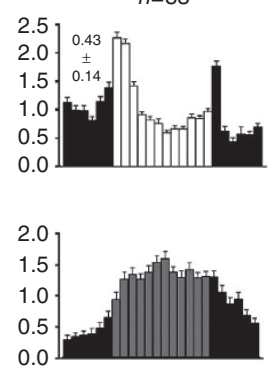
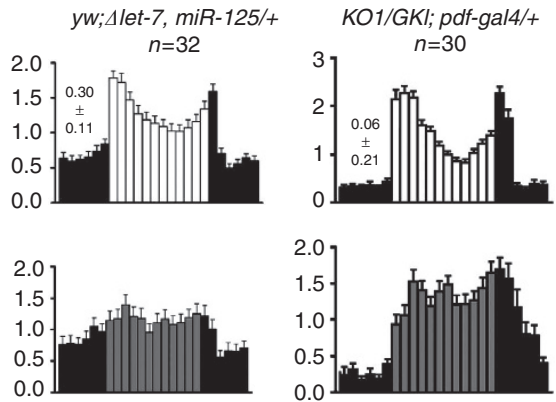

KO1/GKI; pdf-gal4/UAS-let-7 $n=40$
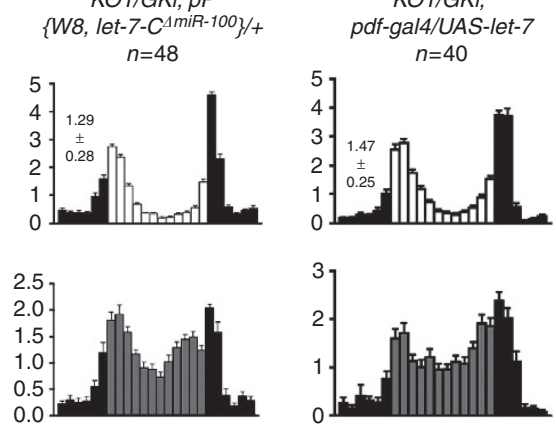

Figure 2 | Genetic ablation of Let-7 causes flies lose morning anticipation. Comparison of the circadian locomotor activity in light/dark cycles of normal let-7-C expression and mutant let-7-C expression flies. Histograms represent the distribution of activity through $24 \mathrm{~h}$, averaged for flies over three LD days and the first day of DD (DD1), and show the activity for each $1 \mathrm{~h}$. Normalized activity profiles are shown. The white and black bars indicate day and night, respectively, while grey and black bars indicate subjective day and night, respectively. Numerical values indicate measures of morning anticipation. Note that genotypes devoid of anticipatory behaviour still respond to light by exhibiting high activity after the light transitions. Total numbers of each genotype are shown in the figure, and each experiment was conducted at least three times. The values were plotted with means \pm s.e.m.

weak) cycling with peak levels in the late day in wild-type flies but not in Clk ${ }^{J r k}$ flies under an LD condition (Fig. 7a). However, prilet-7-C in wild-type flies did not exhibit a daily rhythm compared to mature let-7 under an LD condition (Fig. 7a). This suggests that let-7 cycling may be post-transcriptionally regulated, which is consistent with a previous report ${ }^{38}$.

To investigate whether CLK could directly activate let-7, the let-7-C locus was examined for CLK-biding sites. Since bHLH transcription factors are known to bind to the consensus hexanucleotide sequence E-box (CANNTG) we focused on the various CLK-binding E-boxes described previously ${ }^{39}$. Twentyone E-boxes were found within a $12.5 \mathrm{~kb}$ region surrounding let7-C. Sixteen of these E-boxes are evolutionarily conserved across five Drosophila genomes (D.melanogaster, D.simulans, D.sechellia, D.yakuba and D.erecta). We therefore examined whether CLKCYC can induce gene expression through these E-boxes in Drosophila S2 cell cultures. Cotransfection with CLK did not induce luciferase expression with 7 fragments from the pri-let-7-C promoter region (Supplementary Fig. 6), indicating that pri-let-7$C$ cannot be directly regulated by CLK-CYC through E-boxes, and suggesting that the clock regulates let-7 via an indirect pathway.

A previous publication has shown that the ecdysteroid receptor (EcR) may effectively target the promoter of let-7-C and activate let-7-C $C^{37}$. Thus, it is important to determine if EcR signalling drives let-7-C expression in the adult brain. First, we found that the brain prothoracicotropic hormone (PTTH), an activating hormone for ecdysteroid release, had a daily mRNA rhythm with two peaks at ZT4 and ZT16, and these were blunted in the Clk $k^{J r k}$ mutant in LD (Fig. 7b). In addition, the ptth mRNA oscillation in wild-type and $C l k^{J r k}$ was weakened in DD, although a relatively higher expression at CT4 persisted in wild-type (Fig. 7b). The ptth transcriptional periodicity has previously been shown to be correlated with ecdysteroid titre in Drosophila ${ }^{40}$. To further investigate whether CLK could directly activate $p t t h$, we fused the promoter of ptth from the chr2L: 573711-575711 region with luciferase (ptth-luciferase), and then tested it in S2 cells with timluciferase as a positive control. Results showed that the ptthluciferase was significantly activated when co-transfected with CLK/CYC-expressing plasmids (Fig. 7c), indicating PTTH is a critical target of the clock and may be involved in regulation of the let-7 oscillation. The most prominent peak of ptth mRNA at ZT 4 is not typical of most CLK/CYC-dependent genes, which peak close to ZT16. However, a previous study showed that PTTH cells might be targets of the $\mathrm{s}$ LNv or DN cells and thus stay under control of the master $\operatorname{clock}^{41}$. Our results have also shown that $p t t h$ is a direct target of CLK/CYC. We speculate that the ZT4 and ZT16 peaks are consequences of multiple circadian inputs to the $p t t h$ gene (that is, direct activation by CLK/CYC and inputs resulting from $\mathrm{LNv} / \mathrm{DN}$ signals).

Furthermore, we compared the levels of the 20E steroid hormone (a downstream target of PTTH) at ZT2, 8, 14, 20 between $C l k^{J r k}$ mutants and wild-type flies, and the results showed that $20 \mathrm{E}$ oscillated with a daily rhythm in the wild-type flies but lacked a daily oscillation in the Clk ${ }^{J r k}$ mutant (Fig. 7d). Compared to the $20 \mathrm{E}$ oscillation, the oscillation of let- 7 showed a significant phase delay (Fig. 7a,d).

Moreover, a transgenic line in which each of the ecdysone response elements (EcREs) sequences in the let-7-C locus were deleted $^{37}$ was used to confirm the necessity of EcREs for circadian let-7- $C$ expression. In contrast to the wild-type lac $Z$ reporter, expression of the mutated let-7-Cp12.5kb $\triangle E c R E 1-3:: l a c Z$ reporter was strongly blocked in the pacemaker neurons (Fig. 8a). The ecdysteroid signal is thought to act through a heteromeric receptor composed of the EcR and USP nuclear receptor proteins. So we also blocked ecdysteroid signalling by using the transgenic 
a

b

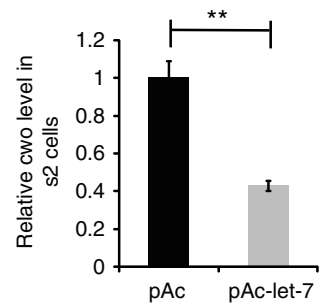

C

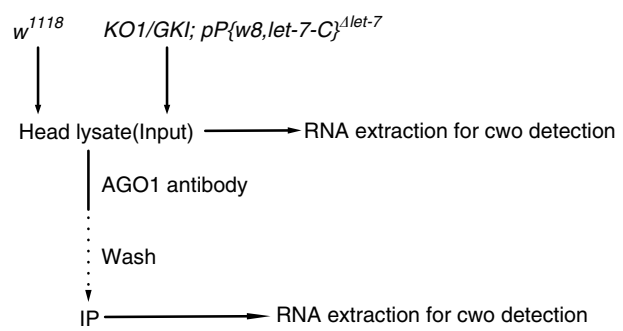

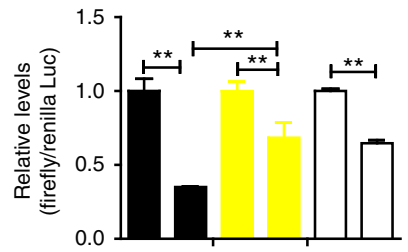

fluc-cwo3'UTR $++-\quad-\quad-$ fluc-cWo3'UTR $(\mathrm{m})--++-$

fluc $-\quad-\quad-\quad-++$

$\begin{array}{rrrrrrr}\text { pAc } & + & - & + & - & + & - \\ \text { Ac-let-7 } & - & + & - & + & - & +\end{array}$
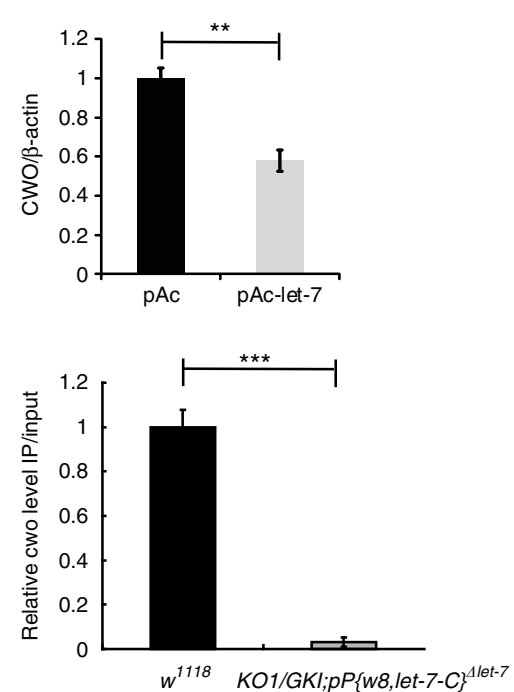

d

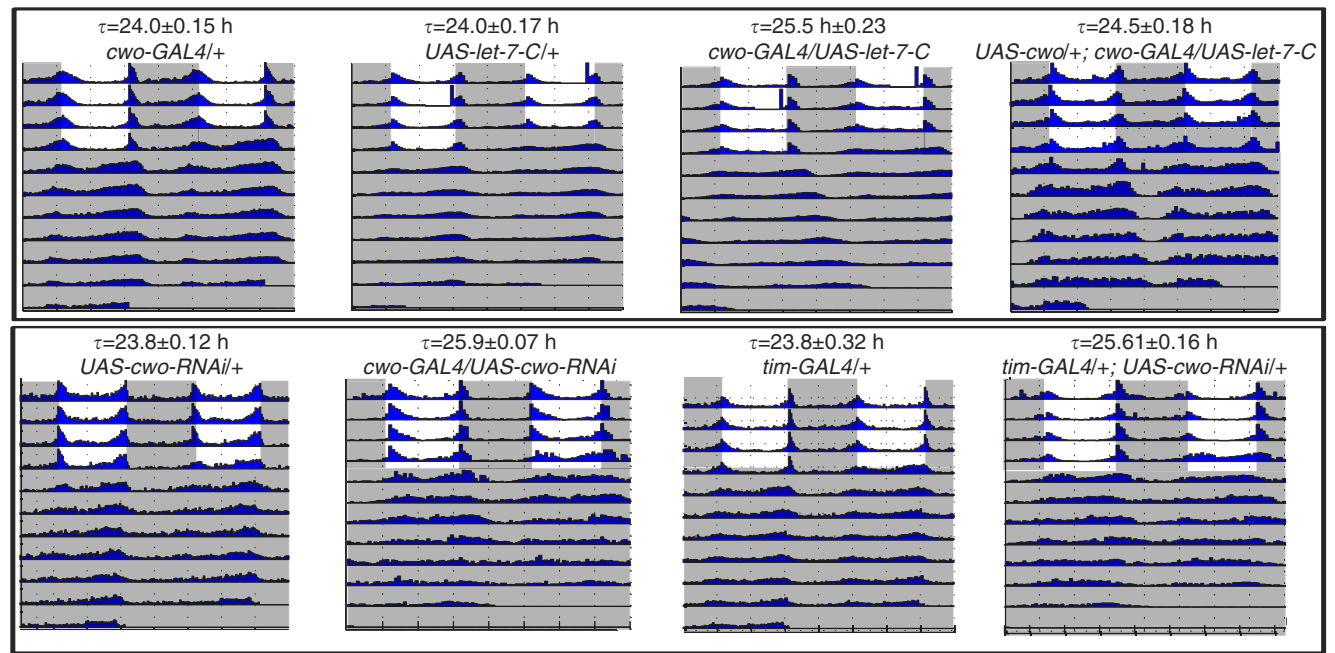

Figure 3 | Cwo is a target of let-7. (a) The native site of the Cwo $3^{\prime} U T R$ sequence containing the let-7 target was amplified and introduced into a firefly luciferase (fluc) reporter vector (pAc-fluc-cwo3'UTR). Sequence with selected point mutations (m) in the Cwo 3'UTR was also constructed as a negative control for the same reporter assays. Luciferase reporter assays were performed after cotransfection with pAc-fluc-cwo3'UTR, pAc-fluc$\mathrm{cwo3}^{\prime} \mathrm{UTR}(\mathrm{m})$ or fluc together with an empty vector $(\mathrm{pAc})$ or a let-7 expression vector (pAc-let-7). In all cases, cotransfection with pCopia-Renilla Luciferase was performed. For each condition, a normalized firefly/renilla luciferase value was plotted with \pm s.e.m. (b) let-7 inhibits CWO protein level by affecting Cwo mRNA in vitro. S2 cells transfected with pAc or pAc-let-7 were lysed and analysed for both Cwo mRNA and CWO protein. Cwo mRNA was detected by quantitative PCR and normalized to rp49 mRNA, and CWO protein was detected by immunoblot with $\beta$-actin as control. Values are reported as fold changes (means \pm s.e.m.) relative to cells transfected with control expression plasmid. (c) Detection of AGO1-associated cwo mRNAs from $w^{1118}$ and $K O 1 / G K I ; p\left\{w 8\right.$,let-7- $\left.C^{\triangle l e t-7}\right\}$ fly heads. Diagram illustrates the AGO1 IP procedure, and the enrichment of cwo was calculated as a ratio between the expression values in the immunoprecipitation and the input fractions, normalized to the value for $w^{1118}$. AGO1-associated cwo mRNA from $w^{1118}$ was significantly higher than AGO1-associated cwo mRNA from KO1/GKl; $p\left\{w 8\right.$,let-7-C $\left.C^{\triangle l e t-7}\right\}$. (d) Overexpression of let-7-C or RNAi knockdown of $c w 0$ with cwo-Gal4 or tim-GAL4 leads to long behavioural periods in DD. The genotypes are indicated on top of the panels. The white and grey parts indicate light and dark, respectively. Expression of Cwo was able to partially rescue the phenotype of let-7-C overexpression in cwo cells (UAS-cwo/ + ; cwo-Gal4/UAS-let-7C) (top panels). Knockdown of Cwo in either cwo- (bottom panels) or tim-expressing (bottom panels) neurons causes long periods, which mimicked the phenotype of let-7-C overexpression. Each experiment was conducted at least three times, and 60-90 flies of each genotype were used for behaviour assays. A significant difference by Student's $t$-test is indicated $\left({ }^{\star \star} P<0.01\right.$ and $\left.{ }^{\star \star \star} P<0.001\right)$. 

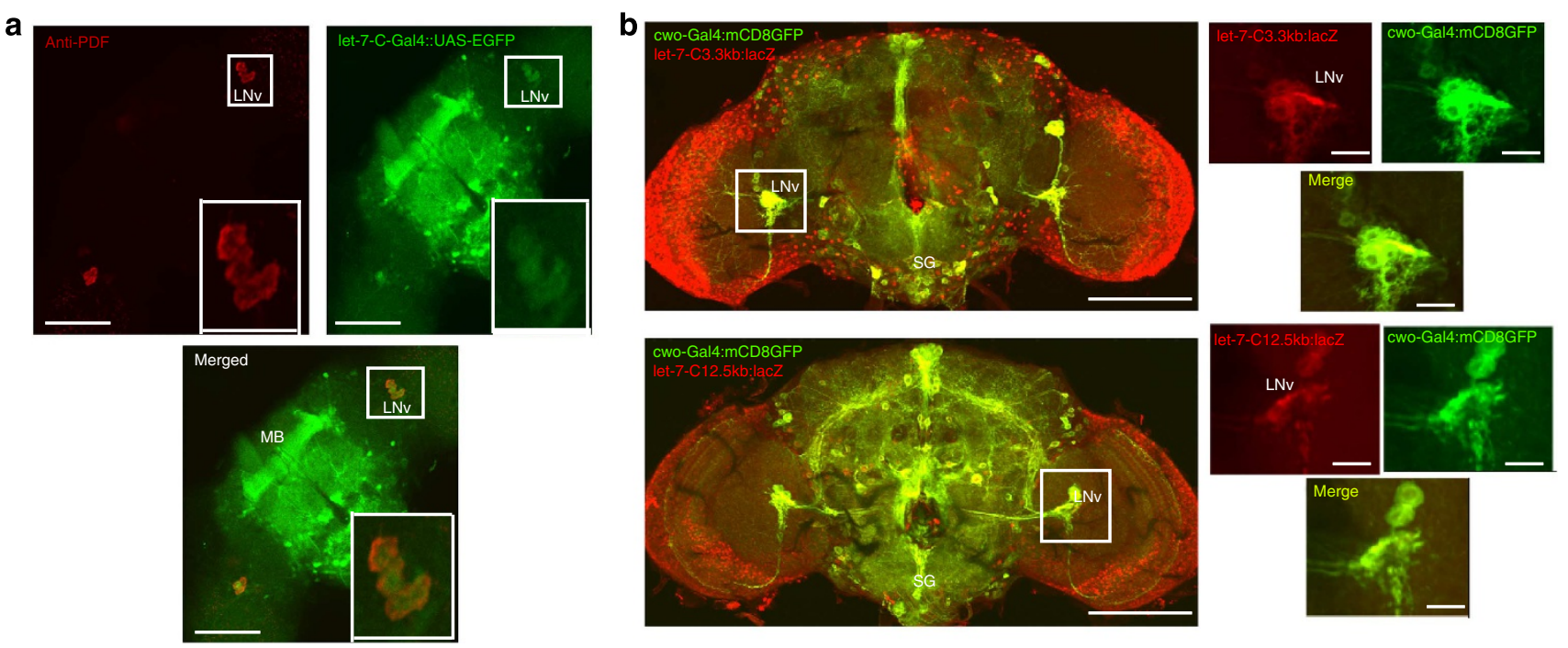

Figure 4 | let-7 is expressed in central clock cells. (a) let-7-C-Gal4 is expressed in LNvs. Three- to five-day-old files expressing enhanced green fluorescent protein were entrained to LD for 3 days. Brains were dissected and stained with anti-PDF (red) antibody at ZT1 on the 3rd day in LD, while GFP was detected directly by its fluorescence. The regions in the boxes are magnified in the panels at the bottom right. MB, mushroom bodies. (b) let-7- $C$ intron 1 transcriptional reporters were used to detect let-7 expression in LNvs. A membrane-targeted mCD8-GFP was expressed in Cwo neurons and detected Cwo expression sites. Brains from the transgenic mutants (cwo-Gal4/UAS-mCD8-GFP;let-7-C3.3kb:lacZ/ + and cwo-Gal4/UAS-mCD8-GFP;let-7$C 12.5 \mathrm{~kb}: \mathrm{lacZ} /+$ ) were dissected and stained with anti- $\beta$-galactosidase at ZT1 on the 3rd day in LD. The region in the box is magnified in the right panels. Each experiment was conducted at least three times, and 10-15 flies of each genotype were used for immunohistochemical assays. Scale bars, $100 \mu \mathrm{m}$ for the whole-mount figure and $20 \mu \mathrm{m}$ for the magnified images.

a

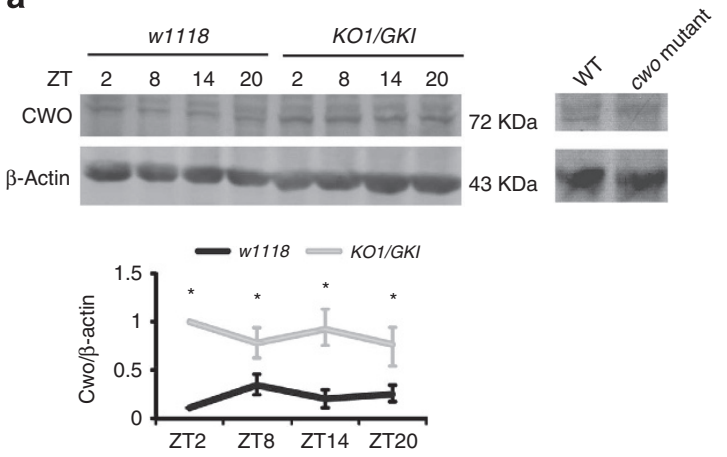

b
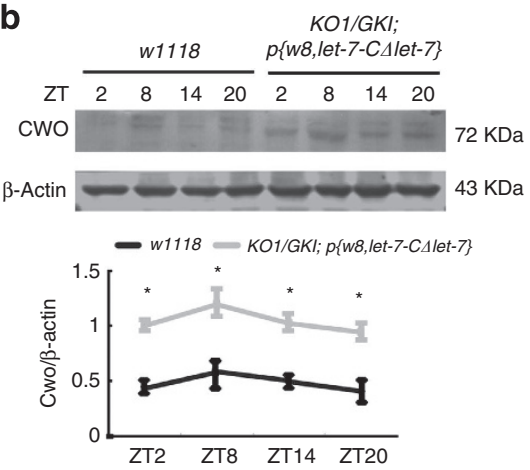

Figure 5 | let-7 affects cwo expression in head. (a) The heads of wild-type ( $w^{1118}$ ), let-7-C mutant (KO1/GKI); and (b) $\triangle l$ let-7 mutant (KO1/GKI;p\{w8,

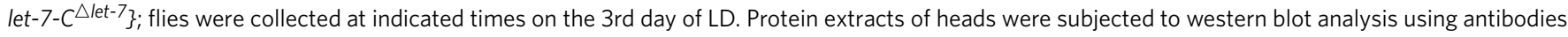
specific for CWO. $\beta$-actin is a loading control. Each experiment was conducted three times, and quantified protein normalized to loading control levels was further normalized to those of the maximum level time point of $K O 1 / G K I$ and $K O 1 / G K l ; p\left\{w 8\right.$,let-7-C $\left.C^{\triangle l e t-7}\right\}$ in each gel. In flies, the homozygous Cwo mutants $\mathrm{CWO}^{\mathrm{e} 04207}$ were used as control. The quantification curves were plotted as the mean \pm s.d. of three independent western blots. A significant difference by Student's $t$-test between wild-type and mutant at the same time point is indicated $\left({ }^{\star} P<0.05\right)$.

hs-Gal4-usp.LBD, in which heat shock-induced expression of this USP dominant-negative form disrupts ecdysteroid signalling during adulthood. This resulted in the absence of let-7 oscillations in the fly head and depressed the daytime levels of let-7 (Fig. 8b). We further tested the morning and evening peaks of activity in $h s$-Gal4-usp.LBD flies under LD conditions. Interestingly, we found that adult flies with ecdysteroidsignalling disruption displayed suppressed morning peak anticipation before lights-on (Fig. 8c) and became largely arrhythmic in DD (Supplementary Table 1), which mimicked the phenotype of let-7 mutants. Furthermore, lack of $20 \mathrm{E}$ signal by inhibiting EcR expression results in decrease of let-7 levels (Supplementary Fig. 7a) and increase of Cwo levels
(Supplementary Fig. 7b), while activation of $20 \mathrm{E}$ signal by promoting ptth expression (Supplementary Fig. 7c) results in decrease of Cwo levels (Supplementary Fig. 7d), and lengthening of circadian period (Supplementary Fig. 7e). All of these findings indicate that $E c R$ signalling is required for oscillations of let-7 expression in the fly head and contributes to adult circadian behaviour.

From all of these data in this study, we conclude that let-7 functionally regulates circadian rhythm via the core clock component $\mathrm{CWO}$, and we find a novel regulatory cycle for the circadian rhythm mechanism. This cycle is regulated by CLOCK/ CYCLE via a pathway involving PTTH, ecdysteroid, its dimeric receptor (EcR/USP), let-7 and CWO (Fig. 9). 


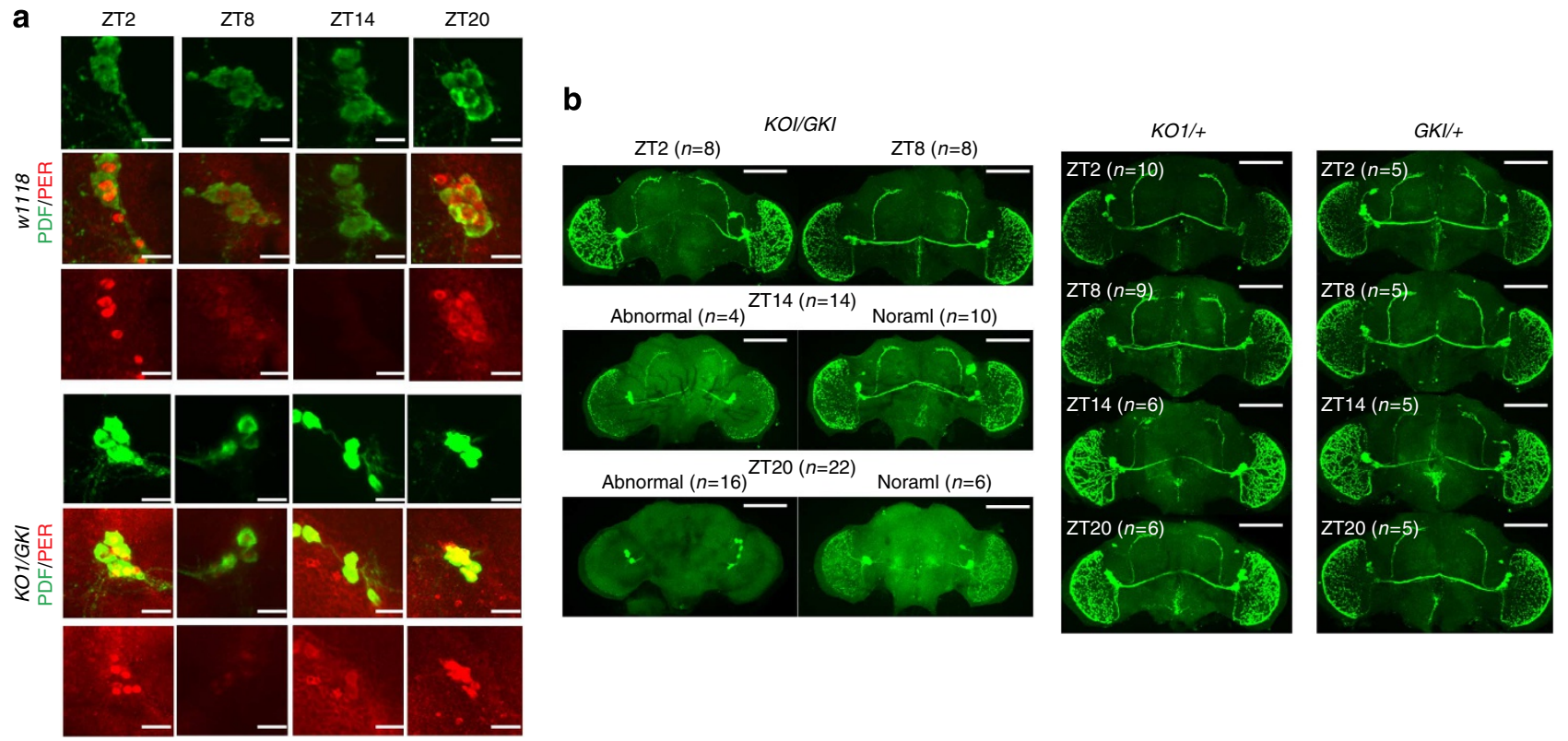

Figure 6 | let-7-C mutant flies display abnormal PER and PDF expression. (a) let-7-C mutant flies show abnormal PER expression in LNvs. The brains of wild-type $\left(w^{1118}\right)$ and let-7-C mutant (KO1/GKI) flies were collected at the indicated times on the third day of LD and stained with PER (red) and PDF (green) antibodies. (b) let-7-C mutant flies show abnormal PDF expression in the projections from LNvs. The brains of control heterozygous files $(K O 1 /+$ and $G K I /+)$ and let-7-C mutant $(K O 1 / G K I)$ flies were collected at the indicated times on the 3rd day of LD and stained with PDF antibodies. Each experiment was conducted at least three times, and 8-22 flies of each genotype were used for immunohistochemical assays. Scale bars, $100 \mu \mathrm{m}$ for the whole-mount figure and $20 \mu \mathrm{m}$ for the magnified images.

\section{Discussion}

A prior study screened for daily rhythms of miRNAs from wild flies and the mutant $c y c^{01}$ by using the microarray method. However, only two Drosophila miRNAs (miR-263a and $m i R-263 b)$ were found to exhibit circadian oscillations ${ }^{29}$. To further investigate miRNAs that exhibit circadian oscillations, Vodala and colleagues used deep sequencing technology to study 18-29 nt RNAs in a $24 \mathrm{~h}$ cycle and found a few miRNAs showed significant oscillations. In this study, we found that let-7 showed significant rhythmic oscillation, consistent with the sequencing data from Vodala ${ }^{31}$. However, some miRNAs have been found to play an important role in circadian regulation even though they do not show significant daily oscillations. For example, two important miRNAs (bantam and miR-279) participate in circadian regulation but are not cycling $28,30,31$.

The let-7-C pri-miRNA consisted of $m i R-100$, let-7 and $m i R$ 125 , and these three miRNAs are produced by differential processing in fly heads. Previous studies showed that miR-100 expression was delayed and its expression level was reduced during development compared to let-7 and miR-125 (ref. 37). In this study, we found that the expression of pri-let-7-C did not exhibit cycling. However, the expression levels of both the mature let-7 and $m i R-125$ exhibited circadian oscillations, with levels increasing from the late night and reaching a peak in the day, while the $m i R$ 100 had no such oscillation (Fig. 7 and Supplementary Fig. 8). Moreover, the expression rhythm of let-7 in the brain is consistent with the expression rhythm of E23-a novel clock gene also induced by ecdyseroid $(20 \mathrm{E})^{42}$ - and was almost antiphase to that of the 20E. This phase delay is probably due to the time required for post-transcriptional processing of let-7.

It is thought that miRNAs mainly function to fine-tune the levels of key proteins. The miRNAs may have a profound effect on the dynamics of regulatory modules by effects on the amplitude of gene expression and alteration of the frequency in the temporal sequence of gene production or delivery ${ }^{43}$. In this study, we found that the CWO levels were higher in let-7-C null mutant flies (Fig. 5). Additionally, our AGO1 immunoprecipitation in $\triangle$ let-7 showed that $c w o$ is a direct target of let-7 in fly heads (Fig. 3). So let-7 probably contributes to the clock rhythms via regulation of $c w o$. From our CWO/let-7 co-staining it is clear that both of them are co-present in only some brain cells (Fig. 4). This probably means that the effects on CWO levels observed in let-7 mutants are partially due to expression of both genes in other tissues, such as eyes. Overexpression of CWO in the cwo-expressing cells didn't generate a let-7 mutant phenotype, but it rescued the rhythm of let-7 overexpression flies (Fig. 3). Moreover, previous studies showed that two copies of Cwo producing overexpression by tim-Gal4 (tim-Gal4/ +; UAS-cwo) generate a modest reduction of rhythmic power ${ }^{12-15}$, which can mimic the let-7 loss-of -function phenotype in this study, indicating that the let-7 mutant phenotype may be due to increased CWO expression. On the contrary, our results show that let-7 overexpression in Cwo cells lengthened circadian period, which is consistent with the effect of $c w o-R N A i$ in both Cwo cells and Tim cells, and it was able to be partially rescued by Cwo overexpression, indicating regulation of Cwo by let-7. CWO function in regulating expression of per/tim has been clear from previous reports ${ }^{12-15}$. In this study, results showed that let-7 is expressed in circadian sLNv neurons, behavioural data showed that the fly's circadian period when let-7-C is overexpressed in endogenous neurons (let-7-C-Gal4/ $+; U A S-l e t-7-C /+$ ) is longer than wild-type $(25.04 \pm 0.26)$, and when it is overexpressed in PDF neurons the period is even longer $(26.12 \pm 0.24$, Table 1$)$. The let-7 mutant phenotype is successfully rescued by let-7 reexpression in PDF neuron (Fig. 2). These results suggest that sLNvs are important circadian neurons for regulation of let-7. Because the UAS-let-7-C seems to have a stronger phenotype that UAS-let-7, we speculate that three miRNAs ( $m i R-125, m i R-100$ and let-7) together may enhance function of let-7, although $m i R$ 125 and miR-100 do not act by themselves. 
a

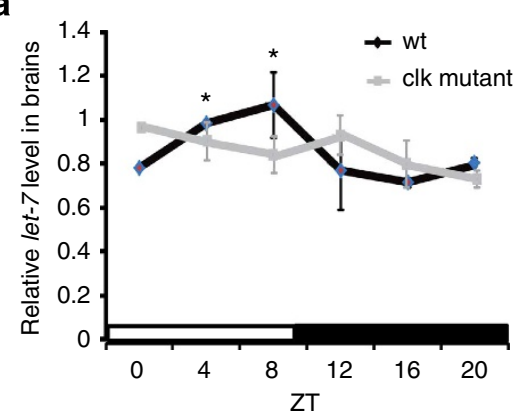

b

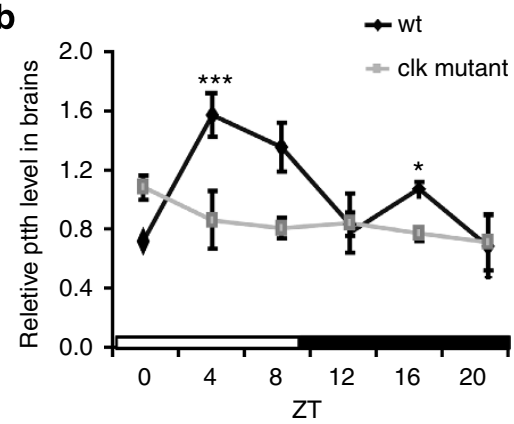

C

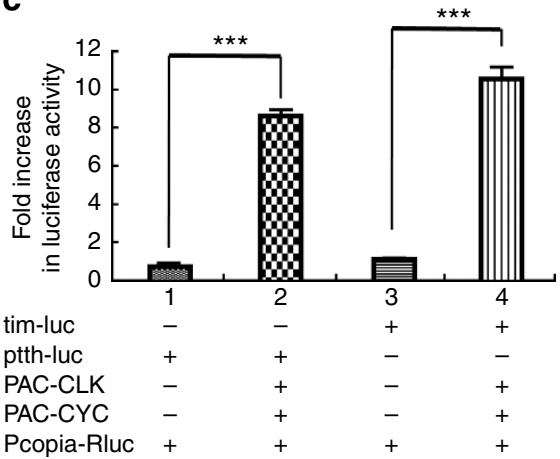

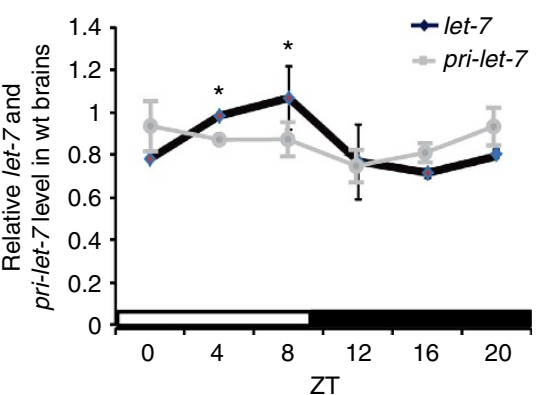

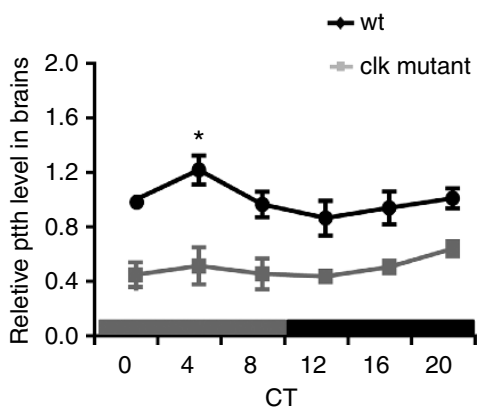

d

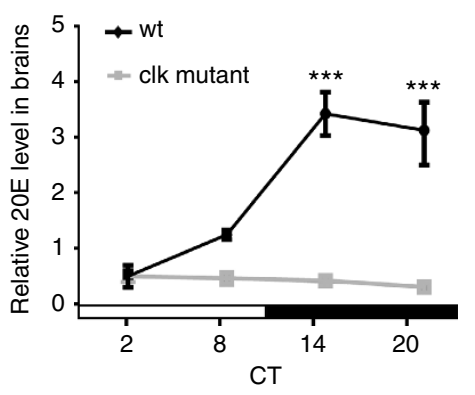

Figure 7 | Let-7 is regulated via PTTH and ecdysteroid signalling. (a) Levels of let-7 are altered in Clk ${ }^{\text {rk }}$ mutant flies. Real time quantitative reverse transcriptase PCR analysis of total RNA prepared from adult brains of the indicated genotypes at the indicated time points. The relative expression levels were normalized to 2S RNA levels and were further normalized to Clk ${ }^{\mathrm{rk}}$ at ZTO (left panel). Each time point was compared to ZTO with Student's $t$-test. ${ }^{\star} P<0.05$. The level of let-7 in wild-type fly brains reached a peak in the day but did not in Clk ${ }^{\text {rk }}$ flies (left panel). The pri-let-7-C didn't show oscillation (right panel). (b) Levels of ptth mRNA are altered in Clk ${ }^{\text {Jrk }}$ mutant flies in LD (left panel) and DD (right panel) conditions. The relative expression levels were normalized to rp49 levels and were further normalized to $C / k^{\text {rrk }}$ at ZTO. Each time point was compared to ZTO with Student's $t$-test. ${ }^{\star} P<0.05$ and ${ }^{\star \star \star} P<0.001$. The levels of ptth in wild-type fly brains had rhythmic peaks at ZT4 and ZT16, while they were eliminated in Clk ${ }^{\text {Jrk }}$ flies. (c) The ptth promoter responds to CLK/CYC. Fold increase in luciferase activity of S2 cells transfected with luciferase reporter constructs containing the promoter of ptth and Clk/Cyc-expressing plasmids. A tim promoter fused to luciferase reporter (tim-luc) was used as positive control. The histogram plot representing fold increase in luciferase activity \pm s.e.m. $(n=3)$ is shown. A significant difference with Student's $t$-test analysis is indicated ( $\left.{ }^{\star \star \star} P<0.001\right)$. (d) Levels of $20 \mathrm{E}$ are under the control of the central clock. Wild-type and $\mathrm{Cl}^{\mathrm{Jrk}}$ mutant fly heads were collected from males at the indicated times for 3 days and $20 \mathrm{E}$ levels were measured using radioimmunoassay (RIA). Three experiments with duplicates for each time point were combined and are presented as the mean \pm s.e.m. The 20 E levels at ZT14 and ZT20 were higher than those of other time points by one-way analysis of variance analysis ( ${ }^{\star \star \star} P<0.001$ ). Each experiment was conducted at least three times, and 25-30 fly heads of each genotype were used for the mRNA and RIA detections.

EcR-mediated signalling in the adult fly nervous system is critical for adult behaviour. Immunohistochemical detection showed that EcR was widely expressed in the adult brain ${ }^{44}$. EcR-mediated signalling in adult flies has an important role in the formation of long-term memory) and sleep regulation ${ }^{44,45}$, and defective long-term memory and sleep in EcR mutants is thought to be caused by insufficient EcR-mediated signalling. Previous studies showed that deletion of EcREs in the pri-let-7-C locus caused a reduction and delay of let-7-C miRNA expression in adult flies and is associated with a let-7-C mutant phenotype ${ }^{37}$. EcREs in intron 1 of the let-7-C locus play an important role in onset and maintenance of let-7-C expression in a tissue-specific manner ${ }^{37}$. In addition, the let-7-C locus was found to be a direct transcriptional target of $20 \mathrm{E}$ because pri-let-7-C RNA could be detected in $\mathrm{KC} 167$ cells 30 min after exposure to $20 \mathrm{E}^{37}$. Taken together, these data strongly suggest that EcR binds to the endogenous let-7-C locus and activates its transcription in response to $20 \mathrm{E}$, and that this transcriptional regulation is required for miRNA let-7 function ${ }^{37}$. In this study, we showed that CLOCK-PTTH-20E-EcR/USP-mediated signalling contributes to circadian regulation, and $20 \mathrm{E}$ mediates circadian expression of let-7 in the adult brain. Moreover, the 20E response is necessary for circadian oscillations in adult pacemaker neurons because the double knockdown of $E c R$ and usp produced an 

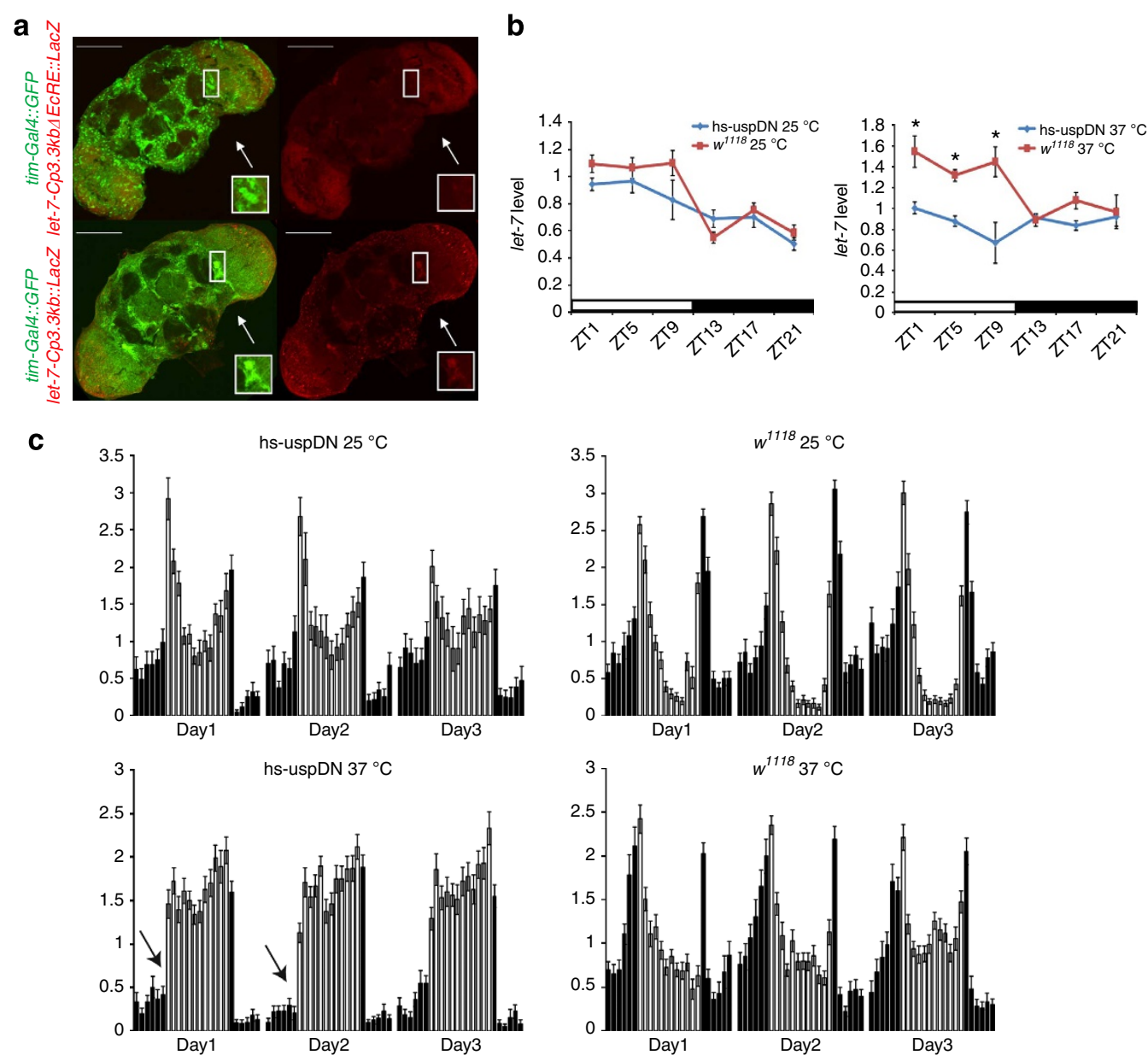

Figure 8 | Ecdysteroid signalling regulates let-7 and rhythmic behaviour. (a) EcR signalling is required for let-7-C expression in fly heads. The transgenic line let-7-Cp12.5kb $\triangle E C R E 1-3:: 1 a c Z^{37}$, in which each of the EcREs sequences was deleted, was coupled with a tim-Gal4::YFP line to monitor the let-7 expression in clock neurons. In contrast to the wild-type lacZ reporter, expression of the mutated reporter was strongly blocked in the pacemaker neurons. Scale bars, $100 \mu \mathrm{m}$ for the whole-mount figure. (b) Blocking ecdysteroid signalling affects let-7 circadian expression in heads. Three-day-old $w^{1118}$ and $w^{1118}$; hs-Gal4-usp.LBD adult flies were heat shocked (starting from ZT12) at $37^{\circ} \mathrm{C}$ for $4 \mathrm{~h}$ and allowed to recover to $25^{\circ} \mathrm{C}$ for $4 \mathrm{~h}$, and this regime was repeated three times per day for 2 days. After heat shock pulses ended for $12 \mathrm{~h}$, samples (30 heads per each sample) were collected at the indicated time points for RNA extraction and real time quantitative reverse transcriptase PCR analysis. The heat shock method was taken from a reference ${ }^{62}$ with some revision. The relative expression level was plotted as mean \pm s.e.m. Levels were normalized relative to those of $h s-G a l 4-u s p . L B D$ flies seen at ZT1. The let-7 level at each time point was compared to ZT21 by one-way analysis of variance with Tukey post-hoc tests ( ${ }^{\star} P<0.05$ ). (c) Blocking ecdysteroid signalling in adult flies results in the loss of the anticipatory morning locomotor activity, thereby mimicking the phenotype of let-7 mutants. The heat shock regime used here was same as that used for panel $\mathbf{b}$. The arrows indicate the loss of the anticipatory morning peaks.

abnormal circadian phenotype ${ }^{42}$. So EcR/USP in circadian neurons receives $20 \mathrm{E}$ and mediates the signalling via let-7, thereby contributing to circadian regulation. However, one complexity to this model is our finding that pri-let-7 mRNA did not exhibit circadian cycling, while let-7 mRNA did cycle. This finding suggests that additional post-transcriptional regulation is necessary to produce cycling of let-7 miRNA, and that pri-let-7 is expressed in additional non-EcR-expressing cells in which it may not be transcribed in a circadian manner and not processed to let-7 (expression in these cells could mask the pri-let7 oscillation in circadian cells).

VRI, a bZIP transcription factor whose expression is controlled by the clock in adult flies ${ }^{46}$, also responds to $20 \mathrm{E}$ both in cell culture and in vivo ${ }^{47}$. Interestingly, the ecdysone-signalling pathway was found to function in the adult circadian system; E23-a novel clock gene that encodes the membrane-bound ABC transporter-was induced by ecdysone, and its knockdown in flies increased expression of vri. E23 and vri responded to both ecdysone and clock signals, whereas E23 protein suppressed the ecdysone response and controlled the circadian oscillation through ecdysone-mediated vri expression ${ }^{42}$. The prothoracic gland in Drosophila larvae and pupae, where ecdysone is secreted, is reported to strongly express clock genes ${ }^{48,49}$. The rhythmic changes in $20 \mathrm{E}$ levels were also regulated by an endogenous circadian oscillator in the haemolymph and testes of adult males in cotton leafworm and in Rhodnius prolixus ${ }^{50,51}$, and $20 \mathrm{E}$ is also present in Drosophila adults ${ }^{52}$. In this study, we found that the PTTH is a direct target of CLK/CYC, most likely because the 2-kb promoter region of ptth contains 10 noncanonical E-box sequences (CANNTG other than CACGTG). Furthermore, circadian oscillations of PTTH, 20E and let-7 in the brain are caused by CLK. Thus, a novel cycle mediated by ecdysteroid was shown to regulate circadian rhythms in this study. The ultimate target of let-7 miRNA is CWO, and this does not appear to cycle by immunoblot analysis of heads (Fig. 5). However, it is likely that CWO does oscillate in a circadian subset of the cells in which 


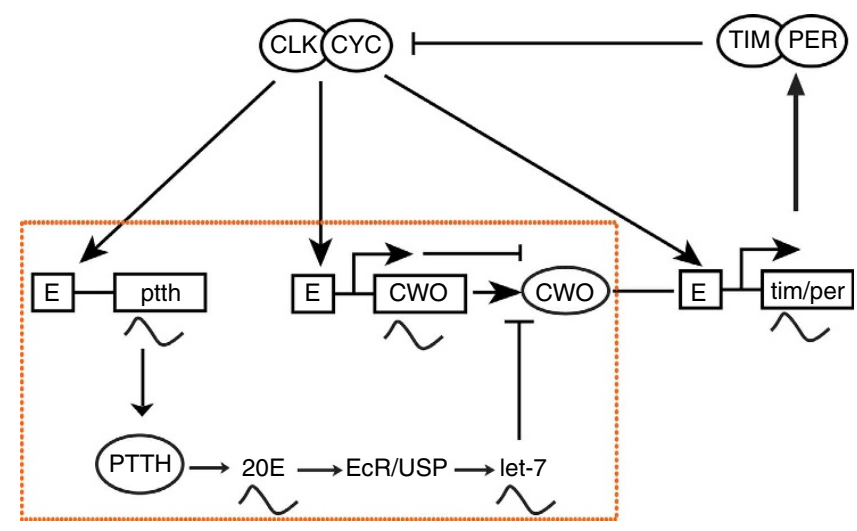

Figure 9 | A regulatory cycle in the circadian clock. A regulatory cycle for the let-7 pathway (orange dotted line), in which let-7 functionally regulates circadian rhythm via core clock component $\mathrm{CWO}$, and let-7 is regulated by CLOCK/CYCLE via a pathway involving PTTH, ecdysteroid and its dimer receptor (EcR/USP). The model for CWO downstream effects in the circadian system is adapted from previous studies ${ }^{12,14}$.

it is expressed, and that constitutive expression in non-circadian cells masks this oscillation in head extracts (note that Fig. 4 shows both let-7 and cwo promoter activity that is more widespread than just circadian cell expression, and Fig. 8 shows that not all let-7 promoter activity is eliminated by deletion of the $E c R$ elements).

miRNAs appear to be major players in the regulation of circadian timekeeping. High throughput sequencing experiments have raised the number of miRNAs to 240 in the Drosophila genome ${ }^{53}$. However, only three miRNAs in Drosophila have been clearly reported to function in regulation of circadian rhythm ${ }^{28,30,31}$. Thus, it may be necessary to perform a genetic screen of individual miRNAs in fly head circadian cells to investigate their potential role in circadian regulation. Recently, a transgenic UAS-miRNA library in Drosophila has been constructed $^{54-56}$. This library circumvents the redundancy issues inherent in loss-of-function screens by facilitating the controlled misexpression of individual miRNAs and is a useful tool to complement loss-of-function approaches. In C.elegans only about $10 \%$ of all miRNA deletions had detectable phenotypic consequences, and Schertel et al. ${ }^{55}$ validated misexpression as an approach to probe miRNA function. Our analysis of let-7 demonstrates the value of this approach for understanding the role of miRNAs in the fly circadian rhythm.

\section{Methods}

Fly strains. Fly strains were maintained on standard molasses-cornmeal-yeast food in a $12 \mathrm{~h}: 12 \mathrm{~h} \mathrm{LD}$ cycle at $25^{\circ} \mathrm{C}$. The fly lines contain: tim-Gal4, pdf-Gal4, cwo-Gal4, tubulin-Gal80ts, tim-Gal4,UAS-2xYFP/Cyo, yw, $w^{1118}, C^{k}{ }^{\text {rrk }}$, hs-Gal4-usp.LBD, UAS-let-7C, UAS-let-7, UAS-miR-125, UAS-mut-let-7, UASEGFP, UAS-cwo-RNAi, let-7-C $C^{G K I} / C y O-T M 6 b$, let-7-C ${ }^{K O 1} / C y O-G F P$, let-7-C ${ }^{K O 1} /$ CyO;p $\left\{w 8\right.$, let-7-C $\left.C^{\triangle l e t-7}\right\}$, let-7-C $C^{K O 1} ; p\left\{w 8\right.$, let-7-C $\left.C^{\triangle m i R-125}\right\} /$ CyO-TM6b, let-7-C ${ }^{K O 1}$, $C y O ; p\{w 8$,let-7-C $\triangle m i R-100\} / T M 6 b, p\left\{w 8\right.$, let-7-C $\left.C^{12.5 k b:: l a c Z}\right\}, p\left\{w 8\right.$,let-7- $\left.C^{3.3 k b:: l a c Z}\right\}$, $p\left\{w 8\right.$, let $\left.-7-C^{12.5 k b \triangle E c R E:: l a c Z}\right\}$

Locomotor behavioural assays. Male fly lines were monitored in a $12 \mathrm{~h}: 12 \mathrm{~h}$ LD cycle at $25^{\circ} \mathrm{C}$ for 3-4 days, followed by 6-7 days in DD using Trikinetics Drosophila Activity Monitors. Activity records were collected in $1 \mathrm{~min}$ bins and analysed using Faas (Fly activity analysis suite) software developed by M. Boudinot and derived from the Brandeis Rhythm Package (http://hawk.bcm.tmc.edu) described by $\mathrm{D}$. Wheeler ${ }^{57}$. Circadian periods were determined by periodogram analysis ${ }^{57}$. A signal-processing toolbox was used to plot actograms ${ }^{58}$. The morning anticipation was determined by assaying of the locomotor activity ${ }^{13}$.

S2 cell culture and luciferase reporter assay. The full length $3^{\prime} \mathrm{UTR}$ of Cwo and an $\sim 500$ bp coding region of let-7 (including pre-miR-125) were amplified by the
PCRusing PrimeSTAR HS DNA Polymerase (TaKaRa). The Cwo $3^{\prime}$ UTR was cloned into a pAc5.1-firefly luciferase-V5-His vector and the let-7 coding region was cloned into a pAc5.1-V5-His vector (Invitrogen). The let-7 'seed'-targeted sequence in the Cwo $3^{\prime}$ UTR was mutated from CTACCTCA to GTTCGTGG using Site-directed, Ligase-Independent Mutagenesis ${ }^{59}$. S2 cells were maintained in $10 \%$ fetal bovine serum (HyClone) Schneider's Insect Medium (Sigma). Cells were seeded in a 12 -well plate. Transfection was performed at $70-90 \%$ confluence with Cellfectin (Invitrogen) according to the manufacturer's instructions. In all experiments, $1 \mu \mathrm{g}$ of miRNA expression plasmid, $100 \mathrm{ng}$ of pCopia-Renilla Luciferase and $100 \mathrm{ng}$ of the luciferase firefly reporter were used. Luciferase assays (Dual Luciferase System, Promega) were performed 2 days after transfection. Renilla luciferase activity provided normalization for firefly luciferase activity.

AG01 immunoprecipitation. $60 \mathrm{fly}$ heads were homogenized in $700 \mu \mathrm{l}$ of lysis buffer (30 mM HEPES KOH at pH 7.4, $100 \mathrm{mM}$ KAcetate, $2 \mathrm{mM}$ MgAcetate, 5\% glycerol, $0.1 \%$ Triton X-100, $1 \mathrm{mM}$ ethylene glycol tetraacetic acid, $5 \mathrm{mM}$ dithio-

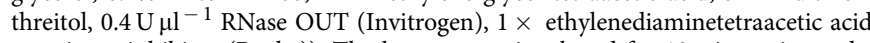
proteinase inhibitor (Roche)). The lysates were incubated for $10 \mathrm{~min}$ on ice and centrifuged at 14,000 r.p.m. for $15 \mathrm{~min}$ at $4{ }^{\circ} \mathrm{C}$. One hundred microliters of the supernatant were used as input. To the rest of the supernatant $(\sim 500 \mu \mathrm{l}), 1 / 10$ volume of anti-AGO1 antibody ( $2.0 \mathrm{ug} \mathrm{ul}^{-1}$, from Miyoshi's lab) was added and incubated at $4{ }^{\circ} \mathrm{C}$ with rotation. After $2 \mathrm{~h}, 100 \mu \mathrm{l}$ of protein $\mathrm{G}$ plus bead slurry (Invitrogen; previously washed with lysis buffer) was added and incubated for $1 \mathrm{~h}$ at $4{ }^{\circ} \mathrm{C}$ with rotation. The beads were then recovered by gentle centrifugation. The beads were washed five times (beads plus $500 \mu$ l of lysis buffer were rotated for 5 min at $4{ }^{\circ} \mathrm{C}$ ), before proceeding to RNA extraction. Total RNA from beads of the $w^{1118}$ wild-type control and $\triangle$ let-7 mutant (KO1/GKI;p $\{w 8$,let-7-C $\triangle$ let-7\}) at the same circadian time (ZT8) was extracted using Trizol.

Real time quantitative reverse transcriptase PCR. Flies were collected at the indicated time points and isolated heads were stored at $-80^{\circ} \mathrm{C}$. Total RNA (including miRNA) was isolated from 30-50 heads with miRcute miRNA isolation kits (TIANGEN). For reverse transcription and real-time PCR (qRT-PCR) of let-7 $m i R-125, m i R-100$ and 2 s rRNA, we used a miRcute miRNA first-stand cDNA synthesis kit and a miRcute miRNA qPCR detection kit (SYBR Green) (TIANGEN). The miRNA-specific forward primers used for qPCR are shown in Supplementary Table 2 . The reverse primer was a primer complementary to the poly(T) adapter that was provided in the miRcute miRNA qPCR detection kit. For reverse transcription and real-time PCR of Cwo, we used the Quantscript RT kit and RealMasterMix (SYBR Green) (TIANGEN). The sequences of primers are shown in Supplementary Table 2. All the experiments were performed in the ABI prism 7500 (Applied Biosystems).

Western blot analysis. Fly heads were homogenized with a pestle, and protein extracts were prepared with radioimmunoprecipitation assay buffer buffer (50 mM Tris- $\mathrm{HCl}$ (pH7.4), $150 \mathrm{mM} \mathrm{NaCl}, 1 \% \mathrm{NP}-40,0.1 \% \mathrm{SDS}$ ). For immunoblot analysis, proteins were transferred to NC membranes (Pall) and incubated with anti-CWO $(1: 1,000$, from Hardin's lab) in blocking solution. Band intensity was calculated and analysed with the Gel-Pro Analyzer 4.0.

Whole-mount brain immunostaining and microsocopy. Adult flies were collected for brain dissection at the indicated times. Brains were fixed for 30-45 $\mathrm{min}$ in $4 \%$ buffered formaldehyde, washed in phosphate-buffered saline ( $\mathrm{pH} 7.4$ ) with $0.3 \%$ Triton X-100 (polybutylene terephthalate), blocked in 5\% goat serum in PBT (phosphate-buffered saline tween-20) for $2 \mathrm{~h}$ and incubated in primary antibody overnight at $4{ }^{\circ} \mathrm{C}$. Indicated dilutions of the following primary antibodies were made in phosphate-buffered saline tween-20: rabbit anti-PER(1:2,000, from Dr Rosbash), rat anti-PDF (1:2,000 from Dr Ceriani) and rabbit anti$\beta$-galactosidase (1:2,000, Promega). After three $15 \mathrm{~min}$ washes, brains were incubated with secondary goat antibodies for $2 \mathrm{~h}$ at room temperature, followed by extensive washes. Secondary antibodies included goat anti-rabbit tetramethylrhodamine and goat anti-rabbit fluorescein isothiocyanate. The GFP and yellow fluorescent protein (YFP) were visualized directly by their excitation. All experiments were imaged on a Nikon ECLIPSE TE2000-E and a Nikon D-ECLIPSE confocal microscope (Nikon, Japan). Confocal images were obtained at an optical section thickness of 1-2 $\mu \mathrm{m}$ and finally analysed with Image J. Staining intensity of PER was calculated and normalized as described ${ }^{60}$.

20E quantification. To determine the titre of $20 \mathrm{E}$ in the heads, we performed a radioimmunoassay developed by David Borst ${ }^{61}$. Briefly, samples were extracted from 50 adult heads by homogenization in $90 \%$ methanol. The homogenates were dried at $70{ }^{\circ} \mathrm{C}$ using a block heater and then dissolved in $200 \mu \mathrm{l}$ borate buffer (0.05 M boric acid; $0.9 \% \mathrm{NaCl} ; 0.1 \%$ gelatin; $0.05 \%$ Triton X-100; $7.7 \mathrm{mM}$ NaAzide; $\mathrm{pH} 8.4$ ) with $\sim 3,000$ disintegrations per minute (DPM) $\left[{ }^{3} \mathrm{H}\right]$ ecdysone and a monoclonal anti-ecdysteroid antibody (diluted 1:12,000). After incubating for $1 \mathrm{~h}$ at room temperature and then overnight at $4{ }^{\circ} \mathrm{C}$ in the refrigerator, the chilled assay tubes was placed on ice for $5 \mathrm{~min}$, and $500 \mu \mathrm{l}$ of stirred, cold dextran-coated charcoal was added $(2.5 \mathrm{mM}$ boric acid; $0.045 \% \mathrm{NaCl} ; 12.5 \mathrm{mg}$ dextran; $38 \mathrm{mM}$ 
ethylenediaminetetraacetic acid; $7.7 \mathrm{mM}$ NaAzide; $0.5 \mathrm{~g}$ charcoal; $\mathrm{pH}$ 8.4) to each tube. After $5 \mathrm{~min}$, the samples were centrifuged at $2,000 \mathrm{~g}$ for $5 \mathrm{~min}$ and the $400 \mu \mathrm{l}$ supernatant was decanted into a new tube. Two millilitres of scintillation cocktail was added, and the each sample was counted in an LS 6500 scintillation counter (Beckman) for $5 \mathrm{~min}$. Calibration curves were generated using the commercially obtained 20E (Sigma, USA).

Statistics analysis. Statistical analysis of two data points was performed with Student's $t$-test. Statistical analysis of multiple data points was performed with oneway analysis of variance with Tukey post-hoc tests.

\section{References}

1. Allada, R. \& Chung, B. Y. Circadian organization of behaviour and physiology in Drosophila. Annu. Rev. Physiol. 72, 605-624 (2010).

2. Hendricks, J. C. et al. Gender dimorphism in the role of cycle (BAML1) in rest, rest regulation, and longevity in Drosophila melanogaster. J. Biol. Rhythm. 18, 12-25 (2003).

3. Lyons, L. C. \& Roman, G. Circadian modulation of short-term memory in Drosophila. Learn. Mem. 16, 19-27 (2009).

4. Sakai, T. \& Ishida, N. Circadian rhythms of female mating activity governed by clock genes in Drosophila. Proc. Natl Acad. Sci. USA 98, 9221-9225 (2001).

5. Cermakian, N. \& Sassone-Corsi, P. Multilevel regulation of the circadian clock. Nat. Rev. Mol. Cell. Biol. 1, 59-67 (2000).

6. Lakin-Thomas, P. L. Circadian rhythms new functions for old clock genes? Trends Genet. 16, 135-142 (2000).

7. Allada, R., White, N. E., So, W. V., Hall, J. C. \& Rosbash, M. A mutant Drosophila homolog of mammalian Clock disrupts circadian rhythms and transcription of period and timeless. Cell 93, 791-804 (1998).

8. Rutila, J. E. et al. CYCLE is a second bHLH-PAS clock protein essential for circadian rhythmicity and transcription of Drosophila period and timeless. Cell 93, 805-814 (1998).

9. Meyer, P., Saez, L. \& Young, M. W. PER-TIM interactions in living Drosophila cells: an interval timer for the circadian clock. Science 311, 226-229 (2006).

10. Cyran, S. A. et al. vrille, Pdp1, and dClock form a second feedback loop in the Drosophila circadian clock. Cell 112, 329-341 (2003).

11. Glossop, N. R. J. et al. VRILLE feeds back to control circadian transcription of Clock in the Drosophila circadian oscillator. Neuron 37, 249-261 (2003).

12. Kadener, S., Stoleru, D., McDonald, M., Nawathean, P. \& Rosbash, M. Clockwork Orange is a transcriptional repressor and a new Drosophila circadian pacemaker component. Genes Dev. 21, 1675-1686 (2007).

13. Lim, C. et al. Clockwork orange encodes a transcriptional repressor important for circadian-clock amplitude in Drosophila. Curr. Biol. 17, 1082-1089 (2007).

14. Matsumoto, A. et al. A functional genomics strategy reveals clockwork orange as a transcriptional regulator in the Drosophila circadian clock. Genes Dev. 21, 1687-1700 (2007).

15. Richier, B., Michard-Vanhee, C., Lamouroux, A., Papin, C. \& Rouyer, F. The clockwork orange Drosophila protein functions as both an activator and a repressor of clock gene expression. J. Biol. Rhythms 23, 103-116 (2008).

16. Fathallah-Shaykh, H. M., Bona, J. L. \& Kadener, S. Mathematical model of the Drosophila circadian clock loop regulation. Biophys. J. 97, 2399-2408 (2009).

17. Nitabach, M. N. \& Taghert, P. H. Organization of the Drosophila circadian control circuit. Curr. Biol. 18, R84-R93 (2008).

18. Grima, B., Chelot, E., Xia, R. \& Francois, R. Morning and evening peaks of activity rely on different clock neurons of the Drosophila brain. Nature 431, 869-873 (2004).

19. Stoleru, D., Peng, Y., Agosto, J. \& Rosbash, M. Coupled oscillators control morning and evening locomotor behaviour of Drosophila. Nature 431, 862-868 (2004).

20. Kojima, S., Shingle, D. L. \& Green, C. B. Post-transcriptional control of circadian rhythms. J. Cell. Sci. 124, 311-320 (2011).

21. Reischl, S. \& Kramer, A. Kinases and phosphatases in the mammalian circadian clock. FEBS Lett. 585, 1393-1399 (2011).

22. Mehta, N. \& Cheng, H. Y. Micro-managing the circadian clock: the role of microRNAs in biological timekeeping. J. Mol. Biol. 425, 3609-3624 (2013).

23. He, L. \& Hannon, G. J. MicroRNAs: small RNAs with a big role in gene regulation. Nat. Rev. Genet. 5, 522-531 (2004).

24. Lewis, B. P., Burge, C. B. \& Bartel, D. P. Conserved seed pairing, often flanked by adenosines, indicates that thousands of human genes are microRNA targets. Cell 120, 15-20 (2005).

25. Du, T. \& Zamore, P. D. microPrimer: the biogenesis and function of microRNA. Development 132, 4645-4652 (2005).

26. Shukla, G. C., Singh, J. \& Barik, S. MicroRNAs: processing, maturation, target recognition and regulatory functions. Mol. Cell Pharmacol. 3, 83-92 (2011).

27. Lai, E. C. Micro RNAs are complementary to $3^{\prime}$ UTR sequence motifs that mediate negative post-transcriptional regulation. Nat. Genet. 30, 363-364 (2002)

28. Kadener, S. et al. A role for microRNAs in the Drosophila circadian clock. Genes Dev. 23, 2179-2191 (2009).
29. Yang, M., Lee, J. E., Padgett, R. W. \& Edery, I. Circadian regulation of a limited set of conserved microRNAs in Drosophila. BMC Genomics 9, 83 (2008).

30. Luo, W. \& Sehgal, A. Regulation of circadian behavioural output via a microRNA-JAK/STAT circuit. Cell 148, 765-779 (2012).

31. Sadanand, V. et al. The oscillating miRNA 959-964 cluster impacts Drosophila feeding time and other circadian outputs. Cell. Metab. 16, 601-612 (2012).

32. Elizabeth, E. C. \& Laura, A. J. Temporal regulation of metamorphic processes in Drosophila by the let-7 and miR-125 heterochronic microRNAs. Curr. Biol. 18, 943-950 (2008).

33. Sokol, N. S., Xu, P., Jan, Y. N. \& Ambros, V. Drosophila let-7 microRNA is required for remodeling of the neuromusculature during metamorphosis. Genes Dev. 22, 1591-1596 (2008)

34. Zhang, L., Lear, B. C., Seluzicki, A. \& Allada, R. The CRYPTOCHROME photoreceptor gates PDF neuropeptide signalling to set circadian network hierarchy in Drosophila. Curr. Bio. 19, 2050-2055 (2009).

35. Grun, D., Wang, Y. L., Langenberger, D., Gunsalus, K. C. \& Rajewsky, N. microRNA target predictions across seven Drosophila species and comparison to mammalian targets. PLoS Comput. Biol. 1, e13 (2005).

36. Ruby, J. G. et al. Evolution, biogenesis, expression, and target predictions of a substantially expanded set of Drosophila microRNAs. Genome Res. 17, 1850-1864 (2007).

37. Chawla, G. \& Sokol, N. S. Hormonal activation of let-7-C microRNAs via EcR is required for adult Drosophila melanogaster morphology and function. Development 139, 1788-1797 (2012).

38. Wulczyn, F. G. et al. Post-transcriptional regulation of the let-7 microRNA during neural cell specification. Faseb J. 21, 415-426 (2007).

39. Abruzzi, K. C. et al. Drosophila CLOCK target gene characterization: implications for circadian tissue-specific gene expression. Genes Dev. 25, 2374-2386 (2011).

40. McBrayer, Z. et al. Prothoracicotropic hormone regulates developmental timing and body size in Drosophila. Dev. Cell 13, 857-871 (2007).

41. Helfrich-Forster, C. Neurobiology of the fruit fly's circadian clock. Genes Brain Behav. 4, 65-76 (2005).

42. Itoh, T. Q., Tanimura, T. \& Matsumoto, A. Membrane-bound transporter controls the circadian transcription of clock genes in Drosophila. Genes Cells 16, 1159-1167 (2011).

43. Nandi, A., Vaz, C., Bhattacharya, A. \& Ramaswamy, R. miRNA-regulated dynamics in circadian oscillator models. BMC Syst. Biol. 3, 45 (2009).

44. Ishimoto, H., Sakai, T. \& Kitamoto, T. Ecdysone signalling regulates the formation of long-term courtship memory in adult Drosophila melanogaster. Proc. Natl Acad. Sci. USA 106, 6381-6386 (2009).

45. Ishimoto, H. \& Kitamoto, T. The steroid molting hormone ecdysone regulates sleep in adult Drosophila melanogaster. Genetics 185, 269-U403 (2010).

46. Blau, J. \& Young, M. W. Cycling vrille expression is required for functional Drosophila clock. Cell 99, 661-671 (1999).

47. Gauhar, Z. et al. Genomic mapping of binding regions for the Ecdysone receptor protein complex. Genome Res. 19, 1006-1013 (2009).

48. Plautz, J. D., Kaneko, M., Hall, J. C. \& Kay, S. A. Independent photoreceptive circadian clocks throughout Drosophila. Science 278, 1632-1635 (1997).

49. Emery, I. F., Noveral, J. M., Jamison, C. F. \& Siwicki, K. K. Rhythms of Drosophila period gene expression in culture. Proc. Natl Acad. Sci. USA 94 4092-4096 (1997).

50. Polanska, M. A., Maksimiuk-Ramirez, E., Ciuk, M. A., Kotwica, J. \& Bebas, P. Clock-controlled rhythm of ecdysteroid levels in the haemolymph and testes, and its relation to sperm release in the Egyptian cotton leafworm, Spodoptera littoralis. J. Insect Physiol. 55, 426-434 (2009).

51. Ampleford, E. J. \& Steel, C. G. Circadian control of a daily rhythm in haemolymph ecdysteroid titre in the insect Rhodnius prolixus (Hemiptera). Gen. Comp. Endocrinol. 59, 453-459 (1985).

52. Handler, A. M. Ecdysteroid titers during pupal and adult development in Drosophila melanogaster. Dev. Biol. 93, 73-82 (1982).

53. Kozomara, A. \& Griffiths-Jones, S. miRBase: integrating microRNA annotation and deep-sequencing data. Nucleic Acids Res. 39, D152-D157 (2010).

54. Bejarano, F. et al. A genome-wide transgenic resource for conditional expression of Drosophila microRNAs. Development 139, 2821-2831 (2012).

55. Schertel, C., Rutishauser, T., Forstemann, K. \& Basler, K. Functional characterization of Drosophila microRNAs by a novel in vivo library. Genetics 192, 1543-1552 (2012).

56. Szuplewski, S. et al. MicroRNA transgene overexpression complements deficiency-based modifier screens in Drosophila. Genetics 190, 617-626 (2012)

57. Klarsfeld, A., Leloup, J.-C. \& Rouyer, F. Circadian rhythms of locomotor activity in Drosophila. Behav. Processes 64, 161-175 (2003).

58. Levine, J. D., Funes, P., Dowse, H. B. \& Hall, J. C. Signal analysis of behavioural and molecular cycles. BMC Neurosci. 3, 1 (2002).

59. Chiu, J., March, P. E., Lee, R. \& Tillett, D. Site-directed, Ligase-Independent Mutagenesis (SLIM): a single-tube methodology approaching $100 \%$ efficiency in 4 h. Nucleic Acids Res. 32, e174 (2004). 
60. Bachleitner, W., Kempinger, L., Wulbeck, C., Rieger, D. \& Helfrich-Förster, C. Moonlight shifts the endogenous clock of Drosophila melanogaster. Proc. Natl Acad. Sci. USA 104, 3538-3543 (2007).

61. Hatle, J. D., Juliano, S. A. \& Borst, D. W. Haemolymph ecdysteroids do not affect vitellogenesis in the lubber grasshopper. Arch. Insect Biochem. Physiol. 52, 45-57 (2003).

62. Kucherenko, M. M., Barth, J., Fiala, A. \& Shcherbata, H. R. Steroid-induced microRNA let-7 acts as a spatio-temporal code for neuronal cell fate in the developing Drosophila brain. EMBO J. 31, 4511-4523 (2012).

\section{Acknowledgements}

We thank J. Price (University of Missouri) and R. Allada (Northwestern University) for the comments on an earlier draft of this manuscript. We are also very grateful to Dr Sokol (Indiana University) for providing most of the let-7 related fly lines and fragments (1-7)luc vectors and thank Dr Rosbash (Brandeis University), Rouyer (Institut de Neurobiologie Alfred Fessard), Ceriani (Fundación Instituto Leloir and Instituto de Investigaciones), Johnston (Columbia University) and Hardin (Texas A\&M University) for providing different types of antibodies, vectors and flies, as well as Dr Levine (University of Toronto) for the signal-processing toolbox to analyse locomotor behaviour. This work was supported by the National Basic Research Program from the Ministry of Science and Technology of the People's Republic of China ('973' Program grant number
2012 CB114100 and 2012CB947600) and the National Natural Science Foundation of China (grant number 31272371) to Z.Z., and Innovation Fund for Graduate Students of China Agricultural University (grant number 2012YJ018) to W.C

\section{Author contributions}

W.C. and Z.Z. conceived and designed the experiments. W.C., Z.L., T.L., R.Z., Y.X., Y.Z., W.B. and D.Z. performed the experiments. W.C. and Z.L analysed the data.

Z.Z. contributed reagents/materials/analysis tools. W.C. and Z.Z. wrote the manuscript.

\section{Additional information}

Supplementary Information accompanies this paper at http://www.nature.com/ naturecommunications

Competing financial interests: The authors declare no competing financial interests.

Reprints and permission information is available online at http://npg.nature.com/ reprintsandpermissions/

How to cite this article: Chen, W. et al. Regulation of Drosophila circadian rhythms by miRNA let-7 is mediated by a regulatory cycle. Nat. Commun. 5:5549 doi: 10.1038/ncomms6549 (2014). 